\title{
Lattice Discrete Particle Modeling of Fiber Reinforced Concrete: Experiments and Simulations
}

\author{
Congrui Jin ${ }^{\mathrm{a}}$, Nicola Buratti ${ }^{\mathrm{b}}$, Marco Stacchini ${ }^{\mathrm{b}}$, Marco Savoia ${ }^{\mathrm{b}}$, Gianluca \\ Cusatis $^{\mathrm{a}, *}$ \\ ${ }^{a}$ Department of Civil and Environmental Engineering, Northwestern University, \\ Evanston, IL 60208, USA. \\ ${ }^{b}$ DICAM-Structural Engineering, University of Bologna, Bologna, Italy
}

\begin{abstract}
Naturally accounting for material heterogeneity, the Lattice Discrete Particle Model (LDPM) is a meso-scale model developed recently to simulate the meso-structure of quasi-brittle materials by a three-dimensional (3D) assemblage of polyhedral particles. A meso-scale constitutive law governs the interaction between adjacent particles and simulates various features of the meso-scale response, including cohesive fracturing, strain softening in tension, strain hardening in compression and material compaction due to pore collapse. LDPM has been extensively calibrated/validated, showing superior capabilities in predicting qualitative and quantitative behavior of concrete. As a natural extension for this discrete model to include the effect of dispersed fibers as discrete entities within the meso-structure, LDPM-F incorporates this effect by modeling individual fibers, randomly placed within the volume according to a given fiber volume fraction. In this investigation, the theoretical basis for LDPM-F is reviewed, and to calibrate/validate the numerical model, an extensive experimental study has been conducted to investigate the mechanical properties of various prismatic specimens containing different types (steel and synthetic) and dosages of fibers. Excellent predictive capability of LDPM-F is demonstrated through a rigorous calibration/validation procedure.
\end{abstract}

Keywords: Fiber Reinforced Concrete, Experiments, Discrete Models 


\section{Introduction}

Concrete is by nature a brittle material that performs well in compression, but is considerably less effective when in tension, and thus reinforcement is often used to absorb these tensile forces so that cracking is either eliminated, e.g., in the case of prestressed members, or, at least, reduced. For many years, steel in the form of bars or mesh has been used as reinforcement for a large variety of structural elements. In recent years, new forms of reinforcement to improve concrete toughness have been developed, such as short randomly distributed fibers of small diameters, made out of steel, plastic, natural materials, recycled reinforcements, etc. [1]. Fiber reinforced concrete, characterized by a significant residual tensile strength in post-cracking regime and enhanced capacity to absorb strain energy due to fiber bridging mechanisms across the crack surfaces, shows a dramatic improvement in service properties over plain concrete. ACI Committee 544 reported that the addition of fibers in a concrete matrix improves some mechanical properties of concrete, including impact strength and flexural toughness [2]. The most beneficial aspects of the use of fibers in concrete structures include durability improvements through reduction in crack width and permeability [3, 4]; potential cost savings where fibers are substituted for labor-intensive steelreinforcing bars [5]; and the mitigation of deterioration owing to shrinkage and the associated early age cracking. Fiber reinforced concrete has found many applications in tunnel linings, bridge decks, airport pavements, slabs on grounds, industrial floors, dams, pipes, fire protection coatings and marine structures. In addition, it can be also used for repair, rehabilitation, strengthening and retrofitting of existing concrete structures [2].

Many numerical models have been developed to simulate the behavior of fiber reinforced concrete at different scales. Most approaches simulate the mechanical response of these composites by means of phenomenological macroscopic models combined with fracture mechanics techniques, such as damage, crack and microplane models [6, 9, 8, 7, 10, 11]. Fanella1 and Krajcinovic developed a nonlinear analytical model based on the principles of the continuum damage mechanics for the stress-strain behavior of fiber reinforced concrete subjected to monotonic compressive and tensile loading [6]. This model was later modified by $\mathrm{Li}$ and $\mathrm{Li}$ to characterize the tensile stress-strain response of the fiber reinforced concrete [9]. Peng and Meyer proposed a simplified continuum damage mechanics model to describe the inelastic behavior of concrete reinforced with randomly distributed short fibers, 
including anisotropic damage, unilateral effect, coupled volumetric and deviatoric inelastic deformation and complicated failure mechanisms [8]. Caner et al. proposed a M7f model for fiber reinforced concretes under static and dynamic loads, which features many improvement over the earlier versions of microplane model, including a more realistic description of the fiber pullout and breakage [7]. Ferreira addresses the use of R-curves to study the fracture behavior of high-strength concrete and steel-fiber-reinforced concrete subjected to cracking in a three-point bending configuration, where the Rcurves are modeled through an effective approach based on the equations of linear-elastic fracture mechanics [10]. Han et al. proposed a constitutive model based on total strain and applied it to simulate structural component tests, which captures fiber reinforced concrete's unique reversed cyclic loading behavior [11].

However, experimental studies on fiber reinforced concrete have confirmed that the mechanisms responsible for the macroscopic mechanical responses mainly involve phenomena that occur at the meso-structural level. The cement fracture is the mechanism that triggers the failure of fiber reinforced concrete, but the subsequent mechanisms leading to complete failure is completely modified by the relative contents of fibers in the composite, and more importantly, by the bond characteristic at the fiber-matrix interface and all the phenomena associated with this effect [12]. Consequently, many discrete frameworks, such as rigid particle and lattice models, have been developed, taking explicitly into account the meso-scale phenomena [13, 14, 15, 16, 17]. A sequential multi-scale homogenization approach has been proposed by Kabele [18], whose framework links analytical and computational models covering scales from micro- to macro-level. Recently, Oliver et al. [19] proposed a two-scale approach in which the macroscopic model, at the structural level, takes into account the meso-structural phenomenon associated with the fibermatrix interface bond/slip process.

In the present work, as first proposed by Cusatis et al. [20, 21, 22], fiber reinforced concrete is analyzed by a multi-scale model, called LDPM-F, in which the fine-scale fiber-matrix interaction is solved independently and the overall response is analyzed in a 3D meso-scale framework based on the recently formulated lattice discrete particle model (LDPM). LDPM is a realistic 3D model of concrete meso-structure developed by Cusatis et al. [23], which has been extensively calibrated/validated under a wide range of quasi-static and dynamic loading conditions, showing superior capabilities in predicting qualitative and quantitative behavior of concrete [25]. LDPM is an improve- 
ment over the confinement-shear lattice model [26, 27], which, along with LDPM, has constitutive laws analogous to those of the microplane model $[28,29]$. As a natural extension for this discrete model to include the effect of dispersed fibers as discrete entities within the meso-structure, LDPM-F incorporates this effect by modeling individual fibers, randomly placed within the framework according to a given fiber volume fraction [30].

LDPM-F has many salient and unique features: (1) a realistic, threedimensional modeling of concrete mesostructure, including a discrete representation of individual fibers randomly distributed therein; (2) a multiscale approach in which the effect of embedded fibers on the structural response is based directly on the micromechanics of the fiber-matrix interaction; (3) the ability to simulate not only tensile fracturing but also multiaxial compressive loading; and (4) true predictive capability demonstrated through a rigorous calibration/validation procedure. In this investigation, the theoretical basis for LDPM-F is reviewed, to calibrate/validate the numerical model, an extensive experimental study has been conducted to investigate the mechanical properties of various prismatic specimens containing different types (steel and synthetic) and dosages of fibers. The predictive capability of LDPM-F is demonstrated through a rigorous calibration/validation procedure.

\section{Review of LDPM-F}

In this section, the theoretical basis for LDPM-F provided by Schauffert and Cusatis [21] is reviewed, and details of the previously established [31] micromechanical fiber pullout model adopted for this study are presented.

\subsection{Extension of LDPM to Include Fiber-Reinforcing Capability}

LDPM simulates concrete mesostructure by considering only coarse aggregate. Particles with assumed spherical shape and in accordance with typical mix designs and granulometric distributions, are introduced randomly into the volume by a procedure which avoids particle overlapping and ensures that all particles are contained within the volume of interest. A Delaunay tetrahedralization of the particle centers, along with the nodes used to describe the external surface of the volume, is used to define the lattice system which represents the mesostructure topology. A 3D domain tessellation, anchored to the Delaunay tetrahedralization, creates a system of polyhedral cells. Each cell contains one aggregate particle (or a surface node), and adjacent cells interact through the triangular facets where they are in contact. 
Fig. 1(a) shows two adjacent particles along with their polyhedral cells and the associated tetrahedron edge. In the LDPM formulation, the interface facets are interpreted as potential crack surfaces. A detailed description of the tessellation procedure and LDPM idealization of concrete mesostructure can be found in Cusatis et al. [23, 25].

The extension to LDPM-F to include fiber-reinforcing capability is performed by inserting individual fibers with randomly generated positions and orientations $[21,22]$. The geometry of an individual fiber is characterized by diameter $d_{f}$, length $L_{f}$, and curvature. In this study, fibers are assumed to be straight, and non-circular cross-sections are simulated through an equivalent diameter calculated as $d_{f}=2\left(A_{f} / \pi\right)^{1 / 2}$, where $A_{f}$ is the fiber cross-sectional area. For a given fiber volume fraction $V_{f}$, the number of fibers $N_{f}$ contained in a concrete volume $V$ is determined by $N_{f}=\left\lceil 4 V_{f} V /\left(\pi d_{f}^{2} A_{f}\right)\right\rceil$, where $\lceil x\rceil$ indicates the ceiling function. The fiber system is then overlapped with the cell system and all the facets intersected by fibers are detected. For each intersection, the shorter and longer fiber lengths, denoted as $L_{s}$ and $L_{l}$, respectively, on each side of the facet, along with the orientations of the fiber and the facet, characterized by unit vectors $\mathbf{n}_{f}$ and $\mathbf{n}$, respectively, are computed. In accordance with the current LDPM formulation, the facet depicted in Fig. 1(b) is the projection of the original tessellation facet onto a plane orthogonal to the associated tetrahedron edge. The projected facet is used in order to avoid non-symmetric behavior for the case of purely tangential movement between two particles and the consequent stress-locking effect which can occur [23].

LDPM describes meso-structure deformation through the adoption of rigid body kinematics. For given displacements and rotations of the particles associated with a given facet, the relative displacement $\llbracket \mathbf{u}_{C} \rrbracket$ at the centroid is used to define measures of strain as $\varepsilon_{N}=\mathbf{n}^{\mathrm{T}} \llbracket \mathbf{u}_{C} \rrbracket / \ell, \varepsilon_{M}=\mathbf{m}^{\mathrm{T}} \llbracket \mathbf{u}_{C} \rrbracket / \ell$, and $\varepsilon_{L}=\mathbf{l}^{\mathrm{T}} \llbracket \mathbf{u}_{C} \rrbracket / \ell$, where $\ell$ is the tetrahedron edge length associated with the facet, and $\mathbf{m}$ and $\mathbf{l}$ are two mutually orthogonal unit vectors tangential to the facet. It can be demonstrated analytically that this measure of strains correspond to the projections onto the facets of the classical strain tensor of continuum mechanics [24]. The strains are then used to compute facet stresses as $\boldsymbol{\sigma}_{c}=\sigma_{N} \mathbf{n}+\sigma_{M} \mathbf{m}+\sigma_{L} \mathbf{l}$ according to the constitutive equation reviewed in the next section. When a facet strains beyond the tensile elastic limit, the meso-scale crack opening can be calculated as $\mathbf{w}=w_{N} \mathbf{n}+w_{M} \mathbf{m}+w_{L} \mathbf{l}$, where $w_{N}=\ell\left(\varepsilon_{N}-\sigma_{N} / E_{N}\right), w_{M}=\ell\left(\varepsilon_{M}-\sigma_{M} / E_{T}\right)$, and $w_{L}=\ell\left(\varepsilon_{L}-\sigma_{L} / E_{T}\right)$ with $E_{N}, E_{T}$ being the elastic normal and tangential LDPM stiffnesses. At 
this point fibers are engaged and equilibrium considerations at the facet level allow for the reasonable assumption of a parallel coupling between the fibers and the surrounding concrete matrix. Total stresses on each LDPM facet are then computed as $\boldsymbol{\sigma}=\boldsymbol{\sigma}_{c}+\left(\sum_{f \in A_{c}} \mathbf{P}_{f}\right) / A_{c}$, where $A_{c}$ is the facet area, $\mathbf{P}_{f}$ is the crack-bridging force for each fiber crossing the given LDPM facet. The following assumptions are made: (1) $\mathbf{P}_{f} \approx \mathbf{0}$ for both $\varepsilon_{N}<0$ and $\mathbf{w}=\mathbf{0}$; and (2) the bridging force depends on the micromechanical crack-bridging mechanisms associated with an increasing meso-scale crack opening, $\mathbf{P}_{f}=\mathbf{P}_{f}(\mathbf{w})$. The crack-bridging force $\mathbf{P}_{f}$ is calculated by analyzing separately the crackbridging effect of one individual fiber on one single meso-scale crack.

\subsection{LDPM Constitutive Law}

A vectorial constitutive law governing the behavior of the material is imposed at the centroid of each facet. The elastic behavior is described by assuming that the normal and shear stresses are proportional to the corresponding strains: $\sigma_{N}=E_{N} \varepsilon_{N} ; \sigma_{M}=E_{T} \varepsilon_{M} ; \sigma_{L}=E_{T} \varepsilon_{L} ;$ where $E_{N}=E_{0}$, $E_{T}=\alpha E_{0}, E_{0}=$ effective normal modulus, and $\alpha=$ shear-normal coupling parameter. $E_{0}$ and $\alpha$ are assumed to be material properties that can be identified from results of experimental tests in the elastic regime. At the macroscopic level, concrete elastic behavior is statistically homogeneous and isotropic. As such, it can be modeled effectively through the classical theory of elasticity characterized by Young's modulus, E, and Poisson's ratio, $\nu$. The relationship between the meso-scale LDPM parameters, $\alpha$ and $E_{0}$, and the macroscopic elastic parameters, $E$ and $\nu$, can be obtained by considering a limiting case in which an infinite number of facets surrounds one aggregate piece. In this case, the LDPM formulation corresponds to the kinematically constrained formulation of the microplane model without deviatoric/volumetric split of the normal strain component, and it can be written as:

$$
E_{0}=\frac{E}{1-2 \nu} \Longleftrightarrow E=\frac{2+3 \alpha}{4+\alpha} E_{0}
$$

and

$$
\alpha=\frac{1-4 \nu}{1+\nu} \Longleftrightarrow \nu=\frac{1-\alpha}{4+\alpha}
$$

For stresses and strains beyond the elastic limit, LDPM mesoscale failure is characterized by three mechanisms as described below.

The first one is fracture and cohesion due to tension and tension-shear. For tensile loading $\left(\varepsilon_{N}>0\right)$, the fracturing behavior is formulated through 
an effective strain and stress:

$$
\varepsilon=\sqrt{\varepsilon_{N}^{2}+\alpha\left(\varepsilon_{M}^{2}+\varepsilon_{L}^{2}\right)}, \quad \sigma=\sqrt{\sigma_{N}^{2}+\left(\sigma_{M}+\sigma_{L}\right)^{2} / \alpha}
$$

which define the normal and shear stresses as

$$
\sigma_{N}=\varepsilon_{N}(\sigma / \varepsilon), \quad \sigma_{M}=\alpha \varepsilon_{M}(\sigma / \varepsilon), \quad \sigma_{L}=\alpha \varepsilon_{L}(\sigma / \varepsilon)
$$

An internal variable $\omega$ characterizes the coupling between normal strain $\varepsilon_{N}$ and total shear strain $\varepsilon_{T}=\sqrt{\varepsilon_{M}^{2}+\varepsilon_{L}^{2}}$, or the normal stress $\sigma_{N}$ and total shear stress $\sigma_{T}=\sqrt{\sigma_{M}^{2}+\sigma_{L}^{2}}$, as:

$$
\tan (\omega)=\varepsilon_{N} / \sqrt{\alpha} \varepsilon_{T}=\sigma_{N} \sqrt{\alpha} / \sigma_{T}
$$

The effective stress $\sigma$ is incrementally elastic $\left(\dot{\sigma}=E_{0} \dot{\varepsilon}\right)$, and must be less than or equal to the strain-dependent boundary:

$$
\sigma_{b t}=\sigma_{0}(\omega) \exp \left[-H_{0}(\omega)\left\langle\varepsilon-\varepsilon_{0}(\omega)\right\rangle / \sigma_{0}(\omega)\right]
$$

where $\langle x\rangle=\max \{x, 0\}$. For pure tensile stress $(\omega=\pi / 2)$, the tensile boundary represents strain-softening with exponential decay, characterized by: (1) the mesoscale tensile strength $\sigma_{t}$; and (2) an initial slope of the exponential decay, or softening modulus $H_{t}$. In order to preserve the correct energy dissipation during mesoscale damage localization, the softening modulus in pure tension is expressed as:

$$
H_{t}=2 E_{0} /\left(l_{t} / l_{e}-1\right)
$$

where $l_{t}=2 E_{0} G_{t} / \sigma_{t}^{2} ; l_{e}$ is the length of the tetrahedron edge under consideration; and $G_{t}$ is the mesoscale fracture energy. For pure shear stress $(\omega=0)$, the effective stress boundary represents perfectly plastic behavior characterized by the mesoscale shear strength $\sigma_{s}$. LDPM provides a smooth transition between pure tension and pure shear. With the ratio of shear strength to tensile strength given as $r_{s t}=\sigma_{s} / \sigma_{t}$, the parabolic variation for the strength is expressed as:

$$
\sigma_{0}(\omega)=\sigma_{t} r_{s t}^{2}\left(-\sin (\omega)+\sqrt{\sin ^{2}(\omega)+4 \alpha \cos ^{2}(\omega) / r_{s t}^{2}}\right) /\left[2 \alpha \cos ^{2}(\omega)\right]
$$

The second physical phenomenon simulated is compaction and pore collapse from compression. For compressive loading $\left(\varepsilon_{N}<0\right)$, the normal stress 
is computed by imposing the inequality $-\sigma_{b c}\left(\varepsilon_{D}, \varepsilon_{V}\right) \leq \sigma_{N} \leq 0$, where $\sigma_{b c}$ is a strain-dependent boundary depending on the volumetric strain $\varepsilon_{V}$ and the deviatoric strain $\varepsilon_{D}$. The volumetric strain is computed at the tetrahedron level as $\varepsilon_{V}=\left(V-V_{0}\right) / 3 V_{0}$, where $V$ and $V_{0}$ are the current and initial volumes of a tetrahedron, respectively. The upper limit of elastic behavior is defined by a volumetric strain value given as: $\varepsilon_{c 0}=\sigma_{c 0} / E_{0}$, where $\sigma_{c 0}$ is a material parameter termed the mesoscale compressive yield stress. Beyond this limit, the compressive boundary is assumed to have an initial linear evolution, modeling pore collapse, up to a volumetric strain value of $\varepsilon_{c 1}=\kappa_{c 0} \varepsilon_{c 0}$. Disregarding the deviatoric-to-volumetric strain ratio, $r_{D V}=\varepsilon_{D} / \varepsilon_{V}$, the stiffness of this linear segment is some fraction of the elastic stiffness and is expressed as $H_{c 0}$. For axial compression with lateral confinement, $r_{D V}$ can significantly affect the response. To simulate the observed horizontal plateau featured by typical experimental data, the stiffness of the pore collapse segment must tend to zero for increasing values of $r_{D V}$. This can be achieved by setting:

$$
H_{c}\left(r_{D V}\right)=H_{c 0} /\left(1+\kappa_{c 2}\left\langle r_{D V}-\kappa_{c 1}\right\rangle\right)
$$

where $H_{c 0}, \kappa_{c 1}$, and $\kappa_{c 2}$ are material parameters. The boundary for the pore collapse phase $\left(\varepsilon_{c 0} \leq-\varepsilon_{V} \leq \varepsilon_{c 1}\right)$ can now be expressed as:

$$
\sigma_{b c}=\sigma_{c 0}+\left\langle-\varepsilon_{V}-\varepsilon_{c 0}\right\rangle H_{c}\left(r_{D V}\right)
$$

Compaction and rehardening occur beyond pore collapse $\left(-\varepsilon_{V} \geq \varepsilon_{c 1}\right)$. In this case one has the following:

$$
\begin{array}{r}
\sigma_{b c}=\sigma_{c 1}\left(r_{D V}\right) \exp \left[\left(-\varepsilon_{V}-\varepsilon_{c 1}\right) H_{c}\left(r_{D V}\right) / \sigma_{c 1}\left(r_{D V}\right)\right] \\
\sigma_{c 1}\left(r_{D V}\right)=\sigma_{c 0}+\left(\varepsilon_{c 1}-\varepsilon_{c 0}\right) H_{c}\left(r_{D V}\right)
\end{array}
$$

Finally, the LDPM constitutive equation simulates friction due to compressionshear. The incremental shear stresses are computed as:

$$
\dot{\sigma}_{M}=E_{T}\left(\dot{\varepsilon}_{M}-\dot{\varepsilon}_{M}^{p}\right), \quad \dot{\sigma}_{L}=E_{T}\left(\dot{\varepsilon}_{L}-\dot{\varepsilon}_{L}^{p}\right)
$$

where $\dot{\varepsilon}_{M}^{p}=\dot{\lambda} \partial \varphi / \partial \sigma_{M}, \dot{\varepsilon}_{L}^{p}=\dot{\lambda} \partial \varphi / \partial \sigma_{L}$, and $\lambda$ is the plastic multiplier with loading-unloading conditions $\varphi \dot{\lambda} \leq 0$ and $\dot{\lambda} \geq 0$. The plastic potential is defined as:

$$
\varphi=\sqrt{\sigma_{M}^{2}+\sigma_{L}^{2}}-\sigma_{b s}\left(\sigma_{N}\right)
$$


where the nonlinear frictional law for the shear strength is assumed to be as follows:

$$
\sigma_{b s}=\sigma_{s}+\left(\mu_{0}-\mu_{\infty}\right) \sigma_{N 0}\left[1-\exp \left(\sigma_{N} / \sigma_{N 0}\right)\right]-\mu_{\infty} \sigma_{N}
$$

where $\sigma_{N 0}$ is the transitional normal stress, and $\mu_{0}$ and $\mu_{\infty}$ are the initial and final internal friction coefficients.

\subsection{Constitutive Law for Fiber-Matrix Interaction}

In the present study, a semi-empirical formulation based on a number of assumptions, including that the fiber is initially straight, elastic, and has negligible bending stiffness, is adopted [31]. Prior to a purely frictional pullout stage, an embedded fiber segment must completely debond from the surrounding matrix. The debonding stage described as a "tunnel-type" cracking process is characterized by two parameters: the bond fracture energy $G_{d}$, and a constant value of frictional stress $\tau_{0}$. The slippage has a critical value $v_{d}$ representing full debonding. For a generic embedment length $L_{e}$, it can be expressed as [32]:

$$
v_{d}=\frac{2 \tau_{0} L_{e}^{2}}{E_{f} d_{f}}+\left(\frac{8 G_{d} L_{e}^{2}}{E_{f} d_{f}}\right)^{1 / 2}
$$

where $E_{f}$ is the modulus of elasticity of the fiber. For the debonding stage $\left(v<v_{d}\right)$, fiber load is given as [32]:

$$
P(v)=\left[\frac{\pi^{2} E_{f} d_{f}^{3}}{2}\left(\tau_{0} v+G_{d}\right)\right]^{1 / 2}
$$

For the pull-out phase $\left(v>v_{d}\right)$, the resistance is entirely frictional and one has [32]:

$$
P(v)=P_{0}\left(1-\frac{v-v_{d}}{L_{e}}\right)\left[1+\frac{\beta\left(v-v_{d}\right)}{d_{f}}\right]
$$

where $P_{0}=\pi L_{e} d_{f} \tau_{0}$, and $\beta$ is a dimensionless coefficient.

In a general situation, the orientation of the embedded segment and the free segment, with associated crack-bridging force $P_{f}$, are different. The original deflection angle between the bridging segment and the embedded end is denoted as $\varphi_{f}$. At the point where the fiber exits the matrix and changes orientation, bearing stresses are created in the underlying matrix. When this localized stress field reaches a sufficient intensity, spalling occurs, and the embedment length of the fiber is reduced by a corresponding length $s_{f}$. The force in the bridging segment experiences a sudden drop, and the 
deflection angle between the two fiber segments is reduced to $\varphi_{f}^{\prime}$. In the current study, the spalling length is obtained by [31]:

$$
s_{f}=\frac{P_{f N} \sin (\theta / 2)}{k_{s p} \sigma_{t} d_{f} \cos ^{2}(\theta / 2)}
$$

where $P_{f N}$ is the normal component of the total force $P_{f}, \sigma_{t}$ is the matrix tensile strength, $k_{s p}$ is a material parameter, and $\theta=\arccos \left(\mathbf{n}_{f}^{\mathrm{T}} \mathbf{n}\right)$.

The snubbing effect is taken into account by assuming that at the point where it exits the tunnel crack, the fiber wraps around the intact matrix in a perfectly flexible manner. The summation of all slip-friction and debonding forces acting parallel to the embedded length is denoted as $P$. The additional friction and bearing forces at the exit point imply that $P_{f}>P$. The following analytical formulation is adopted:

$$
P_{f}=\exp \left(k_{s n} \varphi_{f}^{\prime}\right) P(v)
$$

where $P$ has now been properly expressed as a function of relative fiber slippage $v$, and $k_{s n}$ is a dimensionless material parameter.

The formulations above describe the fiber-matrix interaction only when the load does not cause the rupture of the fiber, it is then necessary to check for each fiber that:

$$
\sigma_{f}=4 P_{f} / \pi d_{f}^{2} \leq \sigma_{u f} e^{-k_{r u p} \varphi_{f}^{\prime}}
$$

where $k_{r u p}$ is a dimensionless material parameter, and $\sigma_{u f}$ is the ultimate tensile strength of the fiber.

For steel fibers, the experimental studies usually provide $\sigma_{u f}$, and sometimes the elastic modulus, usually close to $E_{f}=200 \mathrm{GPa}$.

Consider a fiber, with initial orientation $\mathbf{n}_{f}$, subject to a crack opening of vector $\mathbf{w}$. The tangential component of $\mathbf{w}$ is defined as $w_{T}=\sqrt{w_{M}^{2}+w_{L}^{2}}$. Assuming that the spalling length $s_{f}$ is the same on both sides, the vector for the crack-bridging segment can be computed as $\mathbf{w}^{\prime}=\mathbf{w}+2 s_{f} \mathbf{n}_{f}$. The bridging segment force vector is assumed to be coaxial with the fiber $\mathbf{P}_{f}=P_{f} \mathbf{n}_{f}^{\prime}$, where $\mathbf{n}_{f}^{\prime}=\mathbf{w}^{\prime} /\left\|\mathbf{w}^{\prime}\right\|$. The frictional pulley model applies equally to both the shorter and longer ends, with initial lengths $L_{s}$ and $L_{l}$, relative slippages $v_{s}$ and $v_{l}$, and pullout resisting forces $P_{s}$ and $P_{l}$, respectively. Equilibrium of the fiber bridging segment leads to $P_{f}=\exp \left(k_{s n} \varphi_{f}^{\prime}\right) P_{s}\left(v_{s}\right)=\exp \left(k_{s n} \varphi_{f}^{\prime}\right) P_{l}\left(v_{l}\right)$, where $\varphi_{f}^{\prime}=\arccos \left(\mathbf{n}_{f}^{\mathrm{T}} \mathbf{n}_{f}^{\prime}\right)$, which implies that $P_{s}\left(v_{s}\right)=P_{l}\left(v_{l}\right)$, and compatibility has to be enforced, which is $\left\|\mathbf{w}^{\prime}\right\|=2 s_{f}+v_{s}+v_{l}$. 
As discussed in the sections above, the LDPM-F response depends on two sets of parameters: (a) the LDPM material parameters governing the behavior of plain concrete; and (b) the parameters governing the fiber-matrix interaction model, which needs to be identified by fitting experimental data.

\section{Experimental Data and Test Setup}

The bending test of prismatic specimens is the most popular and widely used experimental technique for assessing the post-cracking behavior of fiber reinforced concrete. It is relatively simple to perform and can be representative of many practical situations. To calibrate/validate the proposed numerical model, an extensive experimental study was conducted to investigate the three-point bending behavior of various prismatic specimens containing different types (steel and synthetic) and dosages of fibers.

The fracture properties of the fiber reinforced concrete were determined according to the European Standard EN14651 [34] and EN14845 [35] which require bending test $(3 \mathrm{PBT})$ on small beam specimens $(150 \times 150 \times 550 \mathrm{~mm})$. The width and depth of test specimens are greater than three times the length of the fibers used in this study, so the preferential fiber alignment effect is minimized [36]. Fracture tests were carried out with a closed-loop hydraulic testing machine by using displacement as control parameter. The experimental results were reported in the form of load versus Crack Mouth Opening Displacement (CMOD) curves, and the latter was measured by means of a resistive displacement transducer positioned astride a notch (having a depth of $25 \mathrm{~mm}$ and a width of $4 \mathrm{~mm}$ ) at mid-span.

In the present work, only a subset of the obtained experimental data was used. In particular, the experimental data on the behavior of the specimens made of plain concrete and concrete containing the fibers were used, as shown in Fig. 2. Fibers SF are hooked steel fibers, and Fibers MS2 are polystyrene fibers. The most important properties of these fibers are listed in Table 1. For each type of fiber, two different dosages were tested, corresponding to the minimum and the maximum dosages suggested by the fiber manufacturers, as shown in Table 2. For each dosage, 7 specimens were tested. Although the experimental setup and test procedure have been described in Ref. [33], the experimental data used in the current study have not been published before.

Existing literature has shown that to improve the compressive concrete strength, flexural strength, and shrinkage, deformed steel fibers are more effective than straight fibers due to mechanical anchorage created by the 
deformed shape [37, 38, 39], and thus it is not surprising that almost all commercially available steel fibers at present are mechanically deformed, such as hemi-circular, hooked, and corrugated fibers [37, 40, 41]. Steel fibers with hooked ends were used in this research. These fibers represent the most widely used steel fibers' geometries used in concrete reinforcement [42].

\section{Model Calibration and Validation}

The proposed numerical model is calibrated by simulating three point bending tests on prismatic notched specimens and by comparing the numerical results with the experimental data. The LDPM concrete parameters are determined by fitting a simulation to the plain concrete response. The fiber model calibration is then performed by fitting a simulation to one of the nonzero $V_{f}$ response curves. For validation purposes, the numerical responses for other fiber contents, determined by altering only the fiber volume fraction $V_{f}$, are then generated, and the results are compared with the experimental curves.

\subsection{Plain Concrete}

In three-point bending experiments, damage typically occurs only in a small region of the beam centered about the mid-span notch. Therefore, in order to reduce the computational cost of the simulations, the mid-span portion was modeled with LDPM-F, and the ends of the beams were modeled with standard elastic finite elements connected to the LDPM-F region through a master-slave algorithm, as shown in Fig. 3(a). The simulation results obtained from the discrete model with LDPM-F defined over the whole specimen are exactly the same as those obtained from the model mixed with LDPM-F in the middle and finite elements at two ends. In Fig. 3(c) the load vs Crack Mouth Opening Displacement (CMOD) curves are shown for plain concrete. In this case the experimental curve (red line) represents the average of two experimental tests, and the numerical curve (green line) represents the average response from two distinct meso-structural models (The figures appear in color in the electronic version of this article). The LDPM concrete parameter values determined by matching the numerical results with experimental data are shown in Table 3 .

\subsection{Steel Fiber Reinforced Concrete}

For steel fiber reinforced concrete, LDPM-F was calibrated by fitting numerical simulation results to the SF-20 $\left(V_{f}=0.26 \%\right)$ curve, and the fiber 
parameters determined from that fit are summarized in Table 4. Fig. 4 shows the specimen geometry and discretization in the numerical simulations, the load vs CMOD curve, and the meso-scale crack patterns at CMOD $=0.8$ $\mathrm{mm}$. In this case the experimental curve (red line) represents the average of seven experimental tests, and the numerical curve (green line) represents the average response from seven distinct meso-structural models.

Numerical prediction of the higher $V_{f}$ response was obtained by changing only $V_{f}$ 's value to $0.45 \%$. The numerical load vs CMOD curves were compared with the experimental curves, as shown in Fig. 5. In this case the numerical results show higher values than the experimental data. The difference between the experimental average and the numerical average, calculated at $\mathrm{CMOD}=0.8 \mathrm{~mm}$, is about $45 \%$. As shown in Fig. 3(d), Fig. 4(c), and Fig. 5(c), for each set of specimen analyzed, the maximum crack opening is about $80 \%$ of the CMOD value.

In order to clarify the discrepancy in the simulation with $V_{f}=0.45 \%$, the fiber arrangement within each specimen was analyzed. It was hoped that such an approach would develop a better understanding of the observed discrepancy, as shown in Fig. 5(b). In the experiments, each specimen was analyzed at the end of the test, and the number of fibers bridging the crack in the three divisions of the cross section, as shown in Fig. 3(b), was counted and the relevant values are reported in Table 5 . To improve reliability of the comparison, seven more numerical simulations were conducted for each case, and the number of fibers bridging the cracks was extracted from the output files of the numerical simulation, as shown in Table 6 .

In Fig. 6(a), at CMOD $=0.8 \mathrm{~mm}$, the load increment due to the effect of fibers, calculated as $100\left(P_{*} / P_{0}-1\right)$, where $P_{*}$ is the load for the fiber reinforced concrete specimens and $P_{0}$ is the average load for the plain concrete specimens, is plotted against the number of active fibers in the lower twothirds of the ligament length, i.e., Bottom + Middle. It can be observed that there is a clear linear relationship between the load increment and the number of active fibers, and that both the experimental data and the numerical results demonstrate a similar trend. However, for the numerical specimens, the range of active fibers increase from [22-39] to [47-70] as the fiber volume fraction nearly doubles from $V_{f}=0.26 \%$ to $V_{f}=0.45 \%$, while for the experimental specimens, the increase of the number of active fibers, from [17-39] to [17-50], is not consistent with the doubling of the fiber volume fraction. It can be concluded that, for the higher value of $V_{f}$, the experimental specimens contain less active fibers than expected, probably due to the inherent 
difficulty of obtaining a uniform fiber distribution during casting. This could be one of the major reasons for the discrepancy observed in the curves shown in Fig. 5(b), and at the same time it highlights the fact that the actual fiber distribution within the specimens is a crucial information needed for the numerical simulation to accurately capture the mechanical behavior of fiber reinforced concrete.

It is worth observing here that the reason that the number of active fibers in the Bottom + Middle part of the specimen was plotted was motivated by analyzing the crack pattern in the numerical models. Fig. 7 shows the colorscale isosurfaces from two typical meso-scale crack openings. Each isosurface corresponds to a different crack opening value. The minimum value that was plotted corresponds to a crack opening magnitude of $2 v_{d}$. This is the overall crack opening associated with a full debonded straight fiber, orthogonal to the crack surface. At this level of crack opening, fibers can be considered fully active. As depicted in Fig. 7, the zone characterized by fully active fibers extends to approximately two-thirds of the ligament length above the notch. The choice of plotting isosurfeces at $\mathrm{CMOD}=0.8 \mathrm{~mm}$ for the comparison is due to the fact that at this value the macroscopic load becomes almost constant.

\subsection{Synthetic Fiber Reinforced Concrete}

For synthetic fiber reinforced concrete, LDPM-F was calibrated by fitting numerical simulation results to the MS2-5 $\left(V_{f}=0.37 \%\right)$ curve, and the fiber parameters determined from that fit are summarized in Table 4 . Fig. 8 shows the specimen geometry and discretization in numerical simulation, the load vs CMOD curve, and the meso-scale crack patterns at CMOD $=0.8 \mathrm{~mm}$. Table 4 shows that, besides $E_{f}$ and $\sigma_{u f}$, only $k_{\text {rup }}$ and $\tau_{0}$ are modified compared to the previous case of steel fibers.

Fig. 9 shows the comparison between the synthetic fiber behavior and the steel fiber behavior during a two way pull-out test. Fig. 9(a) shows that the synthetic fiber undergoes a greater deformation before the complete debonding of the short embedment end since its elastic modulus is much lower than that of the steel fiber. It can be seen that, for the synthetic fiber, the complete debonding of the short embedment end is achieved at $v=0.268$ $\mathrm{mm}$, while for the steel fiber it is achieved at $v=0.086 \mathrm{~mm}$. Fig. 9(b) shows that, due to the differences in elastic modulus and tensile strength, the synthetic fiber undergoes a much lower load level $(0.101 \mathrm{kN})$, before the load drop, than the steel fiber does $(0.474 \mathrm{kN})$. Besides, the synthetic fiber 
load drop is about $0.014 \mathrm{kN}$, whereas the steel fibers load drop is close to $0.071 \mathrm{kN}$.

Numerical prediction of the higher $V_{f}$ response was obtained by changing only the value of $V_{f}$ to $0.74 \%$. The numerical load vs CMOD curves were compared with the experimental curves, as shown in Fig. 10. In this case, LDPM-F is able to predict very accurately the response for the higher fiber content. The error, compared to the experimental results, is only a small percentage at the peak and initial post-peak, and remains less than $20 \%$ even in the far post-peak.

The numerical simulations are further investigated in Fig. 3(d), Fig. 8(c), and Fig. 10(c), where contours of the meso-scale crack openings at CMOD $=0.8 \mathrm{~mm}$, are reported for plain concrete and synthetic fiber reinforced concrete. Due to the presence of the notch and the associated stress concentration, one main macroscopic crack develops in all three cases. This crack, however, is the result of a band of meso-scale cracking characterized by a certain width. This result is in general agreement with the theoretical interpretation provided by $\mathrm{Bazzant}$ and $\mathrm{Oh}$ [43]. For plain concrete, the crack is very localized, and in the vicinity of the notch tip, the crack band width is about $20 \mathrm{~mm}$. This is commensurate with the maximum aggregate size, suggesting very little crack-bridging effect, and is in accordance with the nearly complete loss of load-carrying capacity indicated in Fig. 3(b) for CMOD $=0.8 \mathrm{~mm}$. For synthetic fiber reinforced concrete, the width of the crack band increases significantly with increasing fiber volume fraction. For the case with $V_{f}=0.74 \%$, the crack band width is approximately $75 \mathrm{~mm}$, nearly 3.5 times the maximum aggregate size and nearly twice the fiber length.

Notwithstanding the good agreement between the experimental data and the numerical results for the model validation, the same analysis on the fiber arrangement within each specimen was conducted. Experimental data for the number of fibers bridging the crack in the three divisions of the cross section have been shown in Table 5 . Seven numerical models were analyzed for each case, and the number of fibers bridging the cracks was extracted and shown in Table 6.

The crack pattern in the numerical models was analyzed in Fig. 11, which shows the color-scale isosurfaces for two typical meso-scale crack openings. The minimum crack opening $\left(2 v_{d}\right)$ is $0.300 \mathrm{~mm}$ in this case. As depicted in Fig. 11, the zone characterized by fully active fibers extends to approximately one half of the ligament length above the notch. For this reason the comparison between the experimental data and the numerical results of the load 
increment versus the number of active fibers was now performed by considering two cases: (a) only the numbers of active fibers in the Bottom, as shown in Fig. 6(b); and (b) the numbers of active fibers in the Bottom + Middle, as shown in Fig. 6(c). Again, it can be observed that there is a linear relationship between the load increment and the number of active fibers, and that both the experimental data and the numerical results demonstrate a similar trend.

\subsection{Comparison and Discussion}

The LDPM-F numerical simulations capture many salient features of the experimental results remarkably well: (a) by comparing the load vs CMOD curves for plain concrete and fiber reinforced concrete, the increased loadcarrying capacity of fiber reinforced concrete proportional to fiber volume fraction can be observed during the post-peak period; (b) due to the difference in fibers' mechanical properties, despite the increased fiber volume fraction used in the case of synthetic fibers, the load vs CMOD curves of the synthetic fiber reinforced concrete show much lower load levels during the post-peak compared with steel fiber reinforced concrete; (c) at the peak load, it can be observed that there are no correlations between the load level and the fiber volume fraction since, at that time, the crack opening is so small that the fibers are not active yet and the material response is mainly driven by the concrete matrix, whose maximum strength depends on its random meso-scale structure; and (d) for fiber reinforced concrete, because the fibers' bridging effect leads to the load transmission to the cracked concrete matrix, the size of the crack propagation zone is much greater than that in the case of plain concrete.

However, it can be seen that the numbers of fibers in the numerical models tend to be less scattered than those obtained from the experimental results. This incongruence is caused by the difference in the approaches by which the fibers are arranged within the specimen. During the experiments, the fibers are introduced into the cement matrix non yet aged and then distributed by mixing, whereas during the numerical simulation, LDPM-F uniformly distributed the fibers by a random iterative procedure. To make an efficient and reliable comparison between the experimental and the numerical data, it must be highlighted the importance of recording the exact number of fibers arranged in the volume fraction in which the cracking leads to the specimen failure during the test. It is interesting to note that to predict the evolution of distribution and orientation of fibers in the fiber reinforced self-compacting 
concrete during the casting process, a two-way coupled model for flow of suspension of rigid solid particles in a non-Newtonian fluid with a free surface has been developed recently [44]. This model could be adopted by LDPM-F for the extension to include non-uniform fiber distribution.

\section{Analysis of the Fracture Process Zone}

For cementitious materials, the Fracture Process Zone (FPZ) is defined as the inelastic zone around the crack tip in which energy is dissipated at a certain stage of analysis. The behavior of the FPZ is dominated by complicated mechanisms, such as micro-cracking, crack deflection, bridging, crack face friction, crack tip blunting by voids, and crack branching. Because its length is related to the characteristic length of the cementitious materials, the size, extent, and location of the FPZ have been the subject of countless research efforts for several decades [45, 46, 47, 48]. The understanding of FPZ has been significantly hindered by the fact that, in most experiments, only global quantities, such as the overall strength of the structure, are determined, and local quantities, such as the spatial distribution of dissipated energy and fracture patterns, could not be obtained. Experimentally, local quantities so far have been evaluated by acoustic emission measurements $[49,50,51,52]$, X-ray tomography [53], moiré interferometry [54], and optical microscopy [55]. Digital image correlation techniques have also been applied to study fracture processes in concrete $[56,57,58]$ and other materials $[59,60]$. However, more detailed results are required to fully understand the mechanics of the evolution of fracture processes of concrete, especially fiber reinforced concrete.

The main purpose of this section is to create a 3D numerical methodology to identify the change in fracture characteristics of concrete reinforced with different types of fibers. A somewhat similar analysis, but in $2 \mathrm{D}$, was proposed recently by Grassl et al. [61]. For this purpose, the numerical models analyzed in previous sections for plain concrete and fiber-reinforced concrete were post-processed to understand how the fibers affect the shape and size of the FPZ, as shown in Figs. 12-14, where contours of the meso-scale combined stresses are reported. The combined stress of each facet is defined as the square root of the sum of the squares of the normal stress $\sigma_{N}$, and the two shear stresses $\sigma_{M}$ and $\sigma_{L}$. It should be noted that only the facets that undergo tensile stress, i.e., $\sigma_{N}>0$, are considered, since their progressive failure is responsible for the crack propagation. 
It is well-known that the fracture process zone is subdivided into an inner softening zone and an outer micro-fracture zone. To study the inner zone where the energy dissipation is concentrated, the facets located around it, weakly contributing to the dissipation, are not plotted. Figs. 12-14 are obtained by setting a minimum dissipated power value of $0.001 \mathrm{~J} / \mathrm{s}$ and a minimum combined stress value of $0.2 \mathrm{MPa}$, i.e, the facets with power or stress lower than these values are not shown. During all the tests, the minimum value set for power is less than $1.5 \%$ of the maximum, and the minimum value set for combined stress is less than $5.5 \%$ of the maximum.

\subsection{Plain Concrete}

The evolution of the FPZ for plain concrete is shown in Fig. 12. The minimum power value of $0.001 \mathrm{~J} / \mathrm{s}$ equals to $1.24 \%$ of the maximum at the peak load, which is $0.0803 \mathrm{~J} / \mathrm{s}$, and $0.33 \%$ of the maximum value at CMOD $=0.8 \mathrm{~mm}$ (when the load becomes almost constant), which is $0.3023 \mathrm{~J} / \mathrm{s}$. The minimum stress of $0.2 \mathrm{MPa}$ is $5.21 \%$ of the maximum value at the peak load, which is $3.84 \mathrm{MPa}$, and $4.07 \%$ of the maximum value at CMOD $=0.8 \mathrm{~mm}$, which is $4.92 \mathrm{MPa}$. In the beginning, localized presence of high energy dissipation can be observed just above the notch where the stress is concentrated. With the load increasing, the FPZ size becomes larger and it reaches its maximum width immediately after the peak load. The FPZ width starts to decrease after that because the specimen fails by cracking, and correspondingly, the high stress values move from the notch to the top surface where the concrete matrix has not been damaged yet. In the meantime, the facets localized just above the notch undergo a significant stress drop. At the end of the test, only the facets near to the top surface still contribute to the energy dissipation by enduring combined stress values greater than zero. However, it can be observed that some facets close to the notch, where the concrete matrix has been fully damaged, still have non-zero combined stress values. This apparent inconsistency is caused by the frictional effect between two opposite crack faces while they undergo relative displacement due to not perfectly symmetric specimen deflection.

\subsection{Steel Fiber Reinforced Concrete}

The evolution of the FPZ for steel fiber reinforced concrete with $V_{f}=$ $0.26 \%$ is shown in Fig. 13. The minimum power value of $0.001 \mathrm{~J} / \mathrm{s}$ equals to $1.27 \%$ of the maximum at the peak load, which is $0.0789 \mathrm{~J} / \mathrm{s}$, and $0.13 \%$ of the maximum value at $\mathrm{CMOD}=0.8 \mathrm{~mm}$, which is $0.7672 \mathrm{~J} / \mathrm{s}$. The minimum 
stress of $0.2 \mathrm{MPa}$ is $4.74 \%$ of the maximum value at the peak load, which is $4.22 \mathrm{MPa}$, and $3.74 \%$ of the maximum value at $\mathrm{CMOD}=0.8 \mathrm{~mm}$, which is $5.35 \mathrm{MPa}$. Fig. 13 shows that the FPZ size keeps increasing until the peak load is reached, but different from the previous case of plain concrete, due to the bridging effect between fibers and matrix by which the applied load can be transmitted to the facets even with large crack opening displacements, the facets close to the notch still undergo a certain amount of stress at the end of the test.

\subsection{Synthetic Fiber Reinforced Concrete}

The evolution of the FPZ for synthetic fiber reinforced concrete with $V_{f}=$ $0.74 \%$ is shown in Fig. 14. The minimum power value equals to $1.28 \%$ of the maximum at the peak load, which is $0.0781 \mathrm{~J} / \mathrm{s}$, and $0.23 \%$ of the maximum value at $\mathrm{CMOD}=0.8 \mathrm{~mm}$, which is $0.4376 \mathrm{~J} / \mathrm{s}$. The minimum stress is $3.87 \%$ of the maximum value at the peak load, which is $5.15 \mathrm{MPa}$, and $3.85 \%$ of the maximum value at $\mathrm{CMOD}=0.8 \mathrm{~mm}$, which is $5.20 \mathrm{MPa}$. Similar to the previous cases of plain concrete and steel fiber reinforced concrete, Fig. 14 shows that the FPZ size keeps increasing until the peak load is reached, and then the high stress values move from the notch to the top surface where the concrete matrix has not been damaged yet. Comparison with the cases of steel fiber reinforced concrete shows that the FPZ is much smaller at the end of the test in synthetic fiber reinforced concrete and much less facets experience non-negligible stress levels although higher fiber volume fractions are used. This has been explained by the significantly lower capacity of the synthetic fibers in supporting high stress concentrations, as shown in Fig. 9. For this reason, after an initial phase during which the fibers bridge the cracks close to the notch and transmit the load to the concrete matrix, the fibers' pull-out occurs, leading to a significant drop in the facets' stress levels.

\subsection{Comparison and Discussion}

To compare the FPZ shape and size during the tests for the above discussed cases, Table 7 reported the FPZ width, i.e., the width of the area composed of facets that undergo non-negligible power and stress values, the number of facets in the FPZ, and the value of the total power, both immediately after the peak load at $\mathrm{CMOD}=0.1 \mathrm{~mm}$ when, for each analyzed specimen, the FPZ reaches its maximum width, and at the end of the tests when $\mathrm{CMOD}=1.0 \mathrm{~mm}$ and fibers are fully active. Fig. 15 shows the FPZ 
widths at $\mathrm{CMOD}=0.1 \mathrm{~mm}$ and $\mathrm{CMOD}=1.0 \mathrm{~mm}$ for the five different specimens. Fig. 16 shows the trend of the total power during the tests.

For plain concrete, Table 7 and Fig. 15 show that the FPZ width at $\mathrm{CMOD}=1.0 \mathrm{~mm}$ is much smaller than that at $\mathrm{CMOD}=0.1 \mathrm{~mm}$. As shown in Fig. 16, the total power at the end of the test is about $60 \%$ lower than that at $\mathrm{CMOD}=0.1 \mathrm{~mm}$. This is caused by the concrete matrix failure, which leads to a significant decrease of the number of facets $(68 \%$ less) in which energy is dissipated. The described behavior is representative of the inability of cracked concrete to transfer tensile stress across the crack. The LDPM-F simulation captures this feature very well.

By contrast, for the steel fiber reinforced concrete with $V_{f}=0.26 \%$, the FPZ width at the end of the test is almost the same as immediately after the peak load. The number of facets that make up the FPZ at the end of the test is much larger than that in the case of plain concrete and only $12 \%$ lower than the value at $\mathrm{CMOD}=0.1 \mathrm{~mm}$. As shown in Fig. 16, the trend of the total power of steel fiber reinforced concrete appears very different from that of the plain concrete. The energy dissipation for plain concrete at the end of the test is about one third of the value immediately after the peak load, while for steel fiber reinforced concrete with $V_{f}=0.26 \%$, the total power at the end is still $6 \%$ greater than the value at $\mathrm{CMOD}=0.1 \mathrm{~mm}$. The difference is caused by the fact that in steel fiber reinforced concrete, facets with large crack openings can still support tensile loading due to the fibers' bridging effect.

A similar trend has been observed in steel fiber reinforced concrete with $V_{f}=0.45 \%$. But in this case, because of the higher fiber volume fraction, a larger number of facets in the FPZ and a higher value of the total power are obtained at the end of the test. The number of facets in the FPZ at CMOD $=1.0 \mathrm{~mm}$ is $10 \%$ lower than the value at CMOD $=0.1 \mathrm{~mm}$ while the total power is about $60 \%$ higher.

For synthetic fiber reinforced concrete with $V_{f}=0.45 \%$, the FPZ width at the end of the test $(38.4 \mathrm{~mm})$ is much smaller than that at CMOD $=0.1$ $\mathrm{mm}(84.3 \mathrm{~mm})$. Correspondingly, the number of facets that make up the FPZ and the value of the total power is $62 \%$ smaller and $40 \%$ lower than the values immediately after the peak load, respectively. The specimen with synthetic fiber volume fraction of $0.74 \%$ shows a better behavior due to the higher fiber content, but still, the number of facets at the end of the test is $53 \%$ smaller than that immediately after the peak load, and the value of the total power is $52 \%$ lower. The poor fibers' contribution has been explained 
by the significantly lower capacity of the synthetic fibers in supporting high levels of stress.

From Table 7, it can be seen that the reported values for the five different specimens at $\mathrm{CMOD}=0.1 \mathrm{~mm}$ do not differ significantly from each other. This is because at CMOD $=0.1 \mathrm{~mm}$, the maximum crack openings for fiber reinforced concrete specimens are: $0.079 \mathrm{~mm}$ for SF-20, $0.064 \mathrm{~mm}$ for SF35, $0.082 \mathrm{~mm}$ for MS2-5, and $0.078 \mathrm{~mm}$ for MS2-10, respectively, and so the fibers are not active yet. Recall that the crack openings at complete debonding are $0.094 \mathrm{~mm}$ and $0.300 \mathrm{~mm}$ for steel fibers and synthetic fibers, respectively.

From Fig. 16, it is interesting to observe that the total power of MS2-10 becomes lower than that of MS2-5 after CMOD $=0.7 \mathrm{~mm}$, although MS2-10 has a much higher fiber volume fraction. This could possibly be explained by the randomly distributed fibers and cracks, resulting in fewer active fibers in MS2-10 than in MS2-5.

\section{Characterization Through Round Panel Test}

As reported in previous sections, characteristic values determined from three-point bending tests have a large scatter on the results. It has been shown that this large scatter is due to a nonuniform fiber distribution. Such a scatter becomes particularly large when low fiber volume fractions are used [62]. One of the possible solutions to avoid the large scatter is to increase the cracked area. For this reason, a round panel test, first developed by Bernard [63, 64], was proposed by ASTM [65] in 2004. This type of test has a statically determinate configuration (a round slab having a diameter of 800 $\mathrm{mm}$ and a thickness of $75 \mathrm{~mm}$ with three supports at 120 degrees) where the crack pattern is predictable and the post-cracking material properties can be adequately determined. It appears to be particularly suitable for evaluating the performance of fiber reinforced concrete because the test method involves relatively large deflections. However, handling and placing such a specimen is quite complicated due to the large size and, consequently, high weight (91 $\mathrm{kg}$ ). In addition, standard servo-controlled loading machines may not fit with the geometry of the panel, which is too large for most of them. To meet the need of having a specimen easier to handle, Minelli and Plizzari [66, 67] have proposed the use of a smaller round panel having a diameter of $600 \mathrm{~mm}$ and a depth of $60 \mathrm{~mm}$, with a weight of only $40 \mathrm{~kg}$. Recently, Ciancio et al. [68] have proposed to use even smaller panels having a diameter of $500 \mathrm{~mm}$. It 
has been shown that results from smaller round panels are consistent, reliable and provide a repeatable and predictable crack pattern with a consistently lower dispersion than classical beam tests.

In this section, LDPM-F is calibrated/validated by simulating round panel tests on both large and small specimens and by comparing the numerical results with the experimental data provided by Minelli and Plizzari $[66,67]$.

\subsection{Small Round Panel Tests}

The numerical simulation for small round panel tests was performed on round panels with a diameter of $600 \mathrm{~mm}$ (effective diameter of $550 \mathrm{~mm}$ ) and a thickness of $60 \mathrm{~mm}$, with the load applied to the panels using a rigid cylinder (50 $\mathrm{mm}$ of diameter) above the center of the top surface. The panels were supported by three pivots (40 $\mathrm{mm}$ of diameter), 120 degrees apart radially and positioned $25 \mathrm{~mm}$ from the edge of the panel. The geometry of the specimen is shown in Fig. 17. The translation of the nodes on the bottom surface of the panel in contact with the three pivots is set to be fixed in $z$ direction only, and their rotation is fixed in both $x$ and $y$ directions. The test is displacement controlled by means of the central displacement. The nodes on the top surface of the panel in contact with the loading cylinder are selected, and the loading history is assigned to these nodes.

Since only part of the material parameters are provided in $[66,67]$ and they are the same as those used in previous sections, it is assumed here that the material parameters for concrete and steel fiber used in the experiments $[66,67]$ are the same as those used in previous sections, and so the parameters shown in Table 3 are used in this section for plain concrete, and the parameters for SF shown in Table 1 and Table 4 are used for steel fibers.

The relationship between load and deflection (obtained from three numerical specimens in each case) for different kinds of fiber reinforcement, together with the curves for plain concrete, are shown in Fig. 18. The simulation results have shown that as the panel was loaded, cracks first appeared at the bottom of the panel at its central point, radiated to the edges, and then opened upwards. The typical crack pattern of each round panel was three cracks at angles about 120 degrees, with the panel breaking into 3 pieshaped wedges at large deflections. Fig. 19(a) shows the crack propagation through the round panel obtained from one of the numerical models on plain concrete at $\delta=0.31 \mathrm{~mm}, \delta=0.41 \mathrm{~mm}, \delta=0.48 \mathrm{~mm}$, and $\delta=0.60 \mathrm{~mm}$. Fig. 19(b) shows the crack propagation obtained from one of the numerical 
models on steel fiber reinforced concrete with $V_{f}=0.39$ at $\delta=0.32 \mathrm{~mm}$, $\delta=0.45 \mathrm{~mm}, \delta=0.54 \mathrm{~mm}$, and $\delta=0.63 \mathrm{~mm}$.

Typically, the $P-\delta$ curves of the panels are almost linear up to the peak load. The central deflections at first crack load for both steel fiber reinforced concrete panels are similar, much larger than for the plain concrete. The increase in peak load due to steel fiber reinforcement was not significant. However, it can be seen that the toughness of the concrete panel has been increased dramatically due to steel fiber reinforcement. Fig. 18 shows the comparison between experimental data and numerical results. Note that the use of experimental data is complicated by the fact that the displacement measurement is highly susceptible to inaccuracies caused by several factors, such as the deformation of the supports and the loading platen. To make it simple, the inaccurate part of the displacement is assumed to be linear with the loading, and thus all the experimental data of displacement, $\delta$, are modified as $\delta^{*}=(\delta-P / k)+P / k^{*}$ with $k=50 \mathrm{kN} / \mathrm{mm}$ and $k^{*}=90 \mathrm{kN} / \mathrm{mm}$, intentionally adjusting the slope of the elastic part of the curves to create a match with that of the numerical results. With this adjustment, while not an exact match, the agreement between experimental and numerical curves is quite reasonable.

For plain concrete, Fig. 18 shows three distinct load drops after the peak load, associated with the subsequent formation of the three main cracks, and then a softening behavior by which the load tends to become zero. For steel fiber reinforced concrete, load drops can be observed during the test for all the cases, but the trend is much smoother than in the case of plain concrete, due to the fact that the fibers bridging effect leads to smaller load jumps. In fact, it is difficult to distinguish the three distinct load drops, because when a crack starts to propagate from the panel center, and the load starts to drop, fibers can be intercepted, and the bridging effect leads to load transmission. Nevertheless, in all the cases of steel fiber reinforced concrete, notwithstanding this difference from plain concrete, at the end of the test, three macro-cracks can again be distinguished, but now around them a larger crack propagation zone caused by the fibers bridging effect can be observed.

\subsection{Large Round Panel Tests}

The simulation process and material parameters for large round panel test are exactly the same as those used in small round panel test except for the geometry of the specimen. The numerical simulation for large round panel 
tests was performed on round panels with a diameter of $800 \mathrm{~mm}$ (effective diameter of $750 \mathrm{~mm}$ ) and a thickness of $75 \mathrm{~mm}$, using the same setup as the small round panel test. The panels were supported by three pivots $(40 \mathrm{~mm}$ of diameter), 120 degrees apart radially and positioned $25 \mathrm{~mm}$ from the edge of the panel.

The load-deflection curves for different fiber reinforcements, together with the curves for plain concrete, are shown in Fig. 18. Once again, the load-deflection curves computed by LDPM-F are considered to exhibit a reasonable agreement with those determined experimentally. Fig. 20(a) shows the crack propagation through the round panel bottom surface obtained from one of the numerical models on plain concrete at $\delta=0.31 \mathrm{~mm}, \delta=0.41 \mathrm{~mm}$, $\delta=0.48 \mathrm{~mm}$, and $\delta=0.60 \mathrm{~mm}$. Fig. 20(b) shows the crack propagation obtained from one of the numerical models on steel fiber reinforced concrete with $V_{f}=0.39$ at $\delta=0.48 \mathrm{~mm}, \delta=0.57 \mathrm{~mm}, \delta=1.43 \mathrm{~mm}$, and $\delta=1.90$ mm.

Since for each numerical specimen, three cracks occur, the overall number of fibers involved in the load bearing is more similar among the panels than in case of three point bending test, in which only a single crack occurs in the middle where the notch is located. That is the reason why the load-deflection curve for each round panel shows approximately the same load values in the post-peak softening regime where all the three main cracks have been fully established.

\section{Conclusions}

In this investigation, the fundamental concepts governing the formulation of LDPM-F were reviewed and the mathematical formulation was highlighted. Fiber-matrix interaction is modeled by adapting an established micro-mechanical theory providing the fiber crack-bridging force as a function of the crack opening. For all fibers that intersect an LDPM facet, the crack-bridging forces are added together and assumed to act in parallel with the LDPM constitutive behavior. For calibration/validation purpose, an extensive experimental study was conducted on various prismatic specimens containing different types (steel and synthetic) and dosages of fibers. The numerical simulations conducted on three-point bending tests and round panel tests demonstrated that LDPM-F is able to accurately reproduce the fiber toughening mechanisms, and the associated increases in macroscopic strength and ductility that can be seen in the results of experimental testing while 
keeping the computational cost manageable. In conclusion, the work presented in this paper demonstrates that LDPM-F is an accurate and efficient simulation tool for studying the mechanics of the evolution of fracture processes of fiber reinforced concrete.

\section{Acknowledgment}

This material is partly based upon work supported by the National Science Foundation under grant no. CMMI-1201087. M. Savoia and G. Cusatis conceived and initiated the project. N. Buratti and M. Savoia designed and performed the experimental work. M. Stacchini performed the computation in Section 4 and Section 5. C. Jin performed the computation in Section 6 , analyzed data and wrote the manuscript. G. Cusatis supervised all the computational tasks.

\section{References}

[1] Li, V. C. (2003). "On engineered cementitious composites (ECC): A review of the material and its applications." J. Adv. Concr. Technol., $1(3), 215-230$.

[2] ACI Committee 544 (1996). "State of the art report on fiber reinforced concrete." ACI 544, 1R-96. American Concrete Institute, Detroit.

[3] Belletti, B., Cerioni, R., Meda, A., and Plizzari, G. (2008). "Design aspects on steel fiber-reinforced concrete pavements." J. Mater. Civ. Eng., 20(9), 599-607.

[4] Lepech, M. D., and Li, V. C. (2009). "Water permeability of engineered cementitious composites." Cem. Concr. Compos., 31(10), 744-753.

[5] di Prisco, M., Plizzari, G., and Vandewalle, L. (2009). "Fibre reinforced concrete: New design perspectives." Mater. Struct., 42(9), 1261-1281.

[6] Fanella D., Krajcinovic D. (1985). "Continuum damage mechanics of fiber reinforced concrete." J. Engng. Mech., 111(8), 995-1009.

[7] Caner F. C., Bažant Z. P., Wendner R. (2013). "Microplane model M7f for fiber reinforced concrete." Engineering Fracture Mechanics, 105, 4157 . 
[8] Peng X., Meyer C. (2000). "A continuum damage mechanics model for concrete reinforced with randomly distributed short fibres." Comput. Struct., 78, 505-15.

[9] Li F., Li Z. (2000). "Continuum damage mechanics based modeling of fiber reinforced concrete in tension." Int. J. Solids Struct., 38, 777-793.

[10] Ferreira L. (2007). "Fracture analysis of a high-strength concrete and a high-strength steel-fiber-reinforced concrete." Mech. Compos. Mater., 43, 479-486.

[11] Han T. S., Feenstra P. H., Billington S. L. (2003). "Simulation of highly ductile fiber-reinforced cement-based composite components under cyclic loading." ACI Struct. J., 100(6), 749-57.

[12] Guerrero P., Naaman A. E. (2000). "Effect of mortar fineness and adhesive agents on the pull-out response of steel fibers." ACI Mater. J., 97, $12-20$.

[13] Bolander Jr J. E., Saito S. (1997). "Discrete modeling of short-fiber reinforcement in cementitious composites." Adv. Cem. Based Mater., 6, 76-86.

[14] Li Z., Perez Lara M. A., Bolander J. E. (2006). "Restraining effects of fibers during non-uniform drying of cement composites." Cem. Concr. Res., 36, 1643-52.

[15] Schlangen E., van Mier J. G. M. (1992). "Simple lattice model for numerical simulation of fracture of concrete materials and structures." Mater. Struct., 25, 534-42.

[16] Bolander J. E., Sukumar N. (2005). "Irregular lattice model for quasistatic crack propagation." Phys. Rev. B, 71, 094106.

[17] Bolander J., Choi S., Duddukuri S. (2008). "Fracture of fiber-reinforced cement composites: effects of fiber dispersion." Int. J. Fract., 154, 73-86.

[18] Kabele P. (2007). "Multiscale framework for modeling of fracture in high performance fiber reinforced cementitious composites." Engng. Fract. Mech., 74, 194-209. 
[19] Oliver J., Mora D., Huespe A., Weyler R. (2012). "A micromorphic model for steel fiber reinforced concrete." Int. J. Solids Struct., 49, 29903007.

[20] Cusatis G., Schauffert E. A., Pelessone D., O’Daniel J. L., Marangi P., Stacchini M., Savoia M. (2010). "Lattice discrete particle model for fiber reinforced concrete (LDPM-F) with application to the numerical simulation of armoring systems." Proc., Computational Modelling of Concrete Structures (EURO-C 2010), Taylor and Francis, London.

[21] Schauffert E. A., Cusatis G. (2012). "Lattice discrete particle model for fiber-reinforced concrete. I: Theory." J. Eng. Mech., 138(7), 826-833.

[22] Schauffert E. A., Cusatis G., Pelessone D., O'Daniel J., Baylot J. (2012). "Lattice Discrete Particle Model for fiber reinforced concrete (LDPMF): II Tensile fracturing and multiaxial loading behavior." J. Eng. Mech., 138(7), 834-841.

[23] Cusatis G., Pelessone D., Mencarelli A. (2011). "Lattice discrete particle model (LDPM) for failure behavior of concrete. I: Theory." Cem. Concr. Compos., 33(9), 881-890.

[24] Cusatis G., Zhou X. (2013). "High-order microplane theory for quasibrittle materials with multiple characteristic lengths." ASCE Journal of Engineering Mechanics. 10.1061/(ASCE)EM.1943-7889.0000747.

[25] Cusatis G., Pelessone D., Mencarelli A., Baylot, J. (2011). "Lattice discrete particle model (LDPM) for failure behavior of concrete. II: Calibration and validation." Cem. Concr. Compos., 33(9), 891-905.

[26] Cusatis G., Bažant Z. P., Cedolin L. (2003). "Confinement-shear lattice model for concrete damage in tension and compression. I: Theory." J. Eng. Mech., 129(12), 1439-1448.

[27] Cusatis G., Bažant Z. P., Cedolin L. (2003). "Confinement-shear lattice model for concrete damage in tension and compression. II: Computation and validation." J. Eng. Mech., 129(12), 1449-1458.

[28] Bažant Z. P., Caner F. C., Carol I., Adley M. D., Akers S. A. (2000). "Microplane model M4 for concrete. I: Formulation with workconjugate deviatoric stress." J. Eng. Mech., 126(9), 944-953. 
[29] Di Luzio G., Cusatis G. (2013). "Solidification-microprestressmicroplane (SMM) theory for concrete at early age: theory, validation and application." International Journal of Solids and Structures, 50(6), 957-975.

[30] Smith J., Cusatis G., Pelessone D., Landis E., O’Daniels J., Baylot J. (2014). "Lattice Discrete Particle Modeling of ultra high-performance fiber-reinforced concrete for projectile penetration simulations." International Journal of Impact Engineering, 65, 13-32.

[31] Yang E. H., Wang S., Yang Y., Li, V. C. (2008). "Fiber-bridging constitutive law of engineered cementitious composites." J. Adv. Concr. Technol., 6(1), 181-193.

[32] Lin Z., Kanda T., Li V. C. (1999). "On interface characterization and performance of fiber-reinforced cementitious composites." Concr. Sci. Eng., 1, 173-184.

[33] Buratti N., Mazzotti C., Savoia M. (2011). "Post-cracking behaviour of steel and macro-synthetic fibre-reinforced concretes." Constr. Build Mater., 25, 2713-2722.

[34] UNI EN 14651 (2005). "Test method for metallic fiber concreteMeasuring the flexural tensile strength (limit of proportionally (LOP), residual)." European Committee for Standardization.

[35] UNI EN 14845 (2007). "Test method for fibers concrete Part II: Effect on concrete." European Committee for Standardization.

[36] ASTM C1609/C 1609M-07 (2007). "Standard test method for flexural performance of fiber-reinforced concrete (using beam with third-point loading)." ASTM International, West Conshohocken, PA.

[37] Banthia N., Trottier J. F. (1994). "Concrete reinforced with deformed steel fibers, Part I: Bond-slip mechanisms." ACI Mater. J., 91, 435-446.

[38] Ezeldin S., Lowe S. R. (1991). "Mechanical properties of steel fibre reinforced rapid-set materials." ACI Mater. J., 88(4), 384-9.

[39] Ramakrishnan V., Wu G. Y., Hosalli G. (1989). "Flexural behavior and toughness of fibre reinforced concretes." Transport Res. Rec., 1226, 6977. 
[40] Cunha V. M. C. F., Barros J. A. O., Sena-Cruz J. M. (2010). "Pullout behavior of steel fibers in self-compacting concrete." Journal of Materials in Civil Engineering, 22, 1-9.

[41] Robins P., Austin S., Jones P. (2002). "Pull-out behaviour of hooked steel fibres". Materials and Structures, 35, 434-442.

[42] Laranjeira F., Molins C., Aguado A. (2010) "Predicting the pullout response of inclined hooked steel fibers." Cement and Concrete Research, 40, 1471-1487.

[43] Bažant Z. P., Oh B. H. (1983). "Crack band theory for fracture of concrete." Mater. Structures, RILEM, 16(93), 155-177.

[44] Svec O., Skocek J., Olesen J. F., Stang H. (2012) "Fibre reinforced self-compacting concrete flow simulations in comparison with L-Box experiments using Carbopol." 8th RILEM International Symposium on Fibre Reinforced Concrete, Guimaraes, Portugal.

[45] Cedolin L., Dei Poli S., Iori I. (1983). "Experimental determination of the fracture process zone in concrete." Cement and Concrete Research, $13,557-567$.

[46] Castro-Montero A., Shah S. P., Miller R. A. (1990). "Strain field measurement in fracture process zone." Journal of Engineering Mechanics, $116,2463-2484$.

[47] Maji A. K., Shah S. P. (1988). "Process zone and acoustic emission measurement in concrete." Experimental Mechanics, 28, 27-33.

[48] Hu X., Wittmann F. H. (1990). "Experimental method to determine extension of fracture process zone." Journal of Materials in Civil Engineering, 2, 15-23.

[49] Landis E. N. (1999). "Micro-macro fracture relationships and acoustic emissions in concrete." Construction and Building Materials, 13, 65-72.

[50] Otsuka K., Date H. (2000). "Fracture process zone in concrete tension specimen." Engineering Fracture Mechanics, 65, 111-131. 
[51] Haidar K., Pijaudier-Cabot G., Dube J. F., Loukili A. (2004). "Correlation between internal length, the fracture process zone and size effect in model materials." Materials and Structures, 35, 201-210.

[52] Muralidhara S., Raghu Prasad B. K., Hamid Eskandari, Karihaloo B. L. (2010). "Fracture process zone size and true fracture energy of concrete using acoustic emission." Constr. Build Mater., 24, 479-486.

[53] Landis E. N., Nagy E. N., Keane D. T. (2003). "Microstructure and fracture in three dimensions." Engineering Fracture Mechanics, 70, 911925.

[54] Guo Z. K., Kobayashi A. S., Hawkins N. M. (1993). "Further studies on fracture process zone for mode I concrete fracture." Engng. Fract. Mech., 46, 1041-1049.

[55] Hadjab S. H., Chabaat M., Thimus J.-Fr. (2007). "Use of Scanning Electron microscope and the non-local isotropic damage model to investigate fracture process zone in notched concrete beams." Exp. Mech., 47, 47384 .

[56] Choi S., Shah S. P. (1997). "Measurement of deformations on concrete subjected to compression using image correlation." Experimental Mechanics, 37, 307-313.

[57] Corr D., Accardi M., Graham-Brady L., Shah S. (2007). "Digital image correlation analysis of interfacial debonding properties and fracture behavior in concrete." Engng. Fract. Mech., 74, 109-121.

[58] Huon V., Cousin B., Wattrisse B., Maisonneuve O. (2009). "Investigating the thermo-mechanical behaviour of cementitious materials using image processing techniques." Cem. Concr. Res., 39, 529-536.

[59] Gregoire D., Loh O., Juster A., Espinosa H. D. (2011). "In-situ afm experiments with discontinuous dic applied to damage identification in biomaterials." Experimental Mechanics, 51, 591-607.

[60] Espinosa H. D., Juster A. L., Latourte F. J., Loh O. Y., Gregoire D., Zavattieri P. D. (2011). "Tablet-level origin of toughening in abalone shells and translation to synthetic composite materials." Nature Communications, 2, 173-179. 
[61] Grassl P., Gregoire D., Rojas Solano L., Pijaudier-Cabot G. (2012). "Meso-scale modelling of the size effect on the fracture process zone of concrete." International Journal of Solids and Structures, 9, 1818-1827.

[62] Sorelli L., Meda A., Plizzari G. A. (2005). "Bending and uniaxial tensile tests on concrete reinforced with hybrid steel fibers." ASCE Materials Journal, 15, 519-527.

[63] Bernard E. S. (2000). "Behavior of round steel fiber reinforced concrete panels under point loads." Material and structures, 33, 181-188.

[64] Bernard E. S. (2002). "Correlation in the behavior of fiber reinforced shotcrete beam and panel specimens." Material and structures, 35, 156164.

[65] ASTM C1550-04 (2004). "Standard test method for flexural toughness of fiber reinforced concrete (using centrally loaded round panel)." ASTM International, West Conshohocken, PA, pp. 9.

[66] Minelli F., Plizzari G. A. (2010). "Fiber reinforced concrete characterization through round panel test: part I: experimental study" Fracture Mechanics of Concrete and Concrete Structures-High Performance, Fiber Reinforced Concrete, Special Loadings and Structural Applications. Oh B. H., et al. (eds). pp. 1451.

[67] Minelli F., Plizzari G. A. (2011). "A new round panel test for the characterization of fiber reinforced concrete: a broad experimental study." ASTM J. Testing Evaluat., 39(5), 88997.

[68] Ciancio D., Mazzotti C., Buratti N. (2014) "Evaluation of fibrereinforced concrete fracture energy through tests on notched round determinate panels with different diameters." Construction and Building Materials 52, 86-95. 
Table 1: Mechanical Properties of Fibers Used in the Experiments

\begin{tabular}{lccccccc}
\hline \hline Type & Fiber Code & $L_{f}[\mathrm{~mm}]$ & $d_{f}[\mathrm{~mm}]$ & $L_{f} / d_{f}[-]$ & $E_{f}[\mathrm{GPa}]$ & $\sigma_{u f}[\mathrm{MPa}]$ & Shape \\
\hline Steel & SF & 50 & 1 & 50 & 210 & 1100 & Hooked \\
Polystyrene & MS2 & 40 & 0.83 & 48 & 11.3 & 600 & Hooked \\
\hline
\end{tabular}

Table 2: Fiber Dosage Used in the Experiments

\begin{tabular}{lccc}
\hline \hline Mix Code & Number of Specimens & Dosage $\left[\mathrm{kg} / \mathrm{m}^{3}\right]$ & Volume Fraction [\%] \\
\hline MS2-5 & 7 & 5.0 & 0.37 \\
MS2-10 & 7 & 10.0 & 0.74 \\
SF-20 & 7 & 20.0 & 0.26 \\
SF-35 & 7 & 35.0 & 0.45 \\
\hline
\end{tabular}

Table 3: LDPM Concrete Parameter Values

\begin{tabular}{lcccccc}
\hline \hline$E_{0}[\mathrm{GPa}]$ & $\alpha[-]$ & $\sigma_{t}[\mathrm{MPa}]$ & $l_{t}[\mathrm{~mm}]$ & $G_{t}[\mathrm{~N} / \mathrm{m}]$ & $\sigma_{s} / \sigma_{t}[-]$ & $n_{t}[-]$ \\
\hline 51 & 0.25 & 2.21 & 900 & 43 & 2.5 & 0.2 \\
\hline \hline$\sigma_{c 0}[\mathrm{MPa}]$ & $H_{c 0} / E_{0}[-]$ & $\kappa_{c 0}[-]$ & $\kappa_{c 1}[-]$ & $\kappa_{c 2}[-]$ & $\mu_{0}[-]$ & $\mu_{\infty}[-]$ \\
\hline 195 & 0.2 & 2.7 & 1 & 5 & 0.25 & 0.0 \\
\hline \hline$\sigma_{N 0}[\mathrm{MPa}]$ & $E_{d} / E_{0}[-]$ & $d_{0}[\mathrm{~mm}]$ & $c\left[\mathrm{~kg} / \mathrm{m}^{3}\right]$ & $w / c[-]$ & $n_{F}[-]$ & $\rho\left[\mathrm{kg} / \mathrm{m}^{3}\right]$ \\
\hline 600 & 1 & 10 & 357 & 0.49 & 0.5 & 2400 \\
\hline \hline$a / c[-]$ & $d_{a}[\mathrm{~mm}]$ & \multicolumn{7}{c}{} \\
\hline 5.02 & 22 & & & \\
\hline
\end{tabular}


Table 4: LDPM-F Parameter Values Used in Numerical Simulations

\begin{tabular}{lcccc}
\hline \hline & SF-20 & SF-35 & MS2-5 & MS2-10 \\
\hline$G_{d}[\mathrm{~N} / \mathrm{m}]$ & 5.0 & 5.0 & 5.0 & 5.0 \\
$\tau_{0}[\mathrm{MPa}]$ & 6.0 & 6.0 & 1.5 & 1.5 \\
$\beta[-]$ & 0.0 & 0.0 & 0.0 & 0.0 \\
$k_{s p}[-]^{*}$ & 6.2 & 6.2 & 6.2 & 6.2 \\
$k_{\text {sn }}[-]$ & 0.5 & 0.5 & 0.5 & 0.5 \\
$\sigma_{u f}[\mathrm{MPa}]$ & 1100 & 1100 & 600 & 600 \\
$k_{\text {rup }}[-]$ & 0.0 & 0.0 & 0.3 & 0.3 \\
$E_{f}[\mathrm{GPa}]$ & 210 & 210 & 11.3 & 11.3 \\
\hline
\end{tabular}

*In numerical simulation, a simplified procedure has been adopted. Most studies provide the macroscopic compressive strength $\sigma_{c}^{\prime}$ of the concrete or mortar being investigated. Using a mid-range estimate, it is assumed that $\sigma_{t}^{\prime}=\sigma_{c}^{\prime} / 10$, which allows for the approximate calculation of $k_{s p} \sigma_{t}$ as $6.2 \sigma_{c}^{\prime} / 10 \approx 150 \mathrm{Mpa}$. 
Table 5: Numbers of the Fibers Bridging the Cracks in Three Divisions of the Cross Section

\begin{tabular}{lccccccc}
\hline \hline Experiments on SF-20 & & & & & & & \\
\hline Specimen & $\# 1$ & $\# 2$ & $\# 3$ & $\# 4$ & $\# 5$ & $\# 6$ & $\# 7$ \\
Bottom & 8 & 10 & 22 & 18 & 12 & 15 & 18 \\
Middle & 9 & 15 & 17 & 9 & 11 & 13 & 14 \\
Top & 15 & 16 & 23 & 4 & 13 & 24 & 25 \\
\hline \hline Experiments on SF-35 & & & & & & & \\
\hline Specimen & $\# 1$ & $\# 2$ & $\# 3$ & $\# 4$ & $\# 5$ & $\# 6$ & $\# 7$ \\
Bottom & 12 & 17 & 15 & 15 & 19 & 15 & 28 \\
Middle & 5 & 28 & 12 & 15 & 15 & 12 & 22 \\
Top & 19 & 12 & 21 & 22 & 14 & 27 & 25 \\
\hline \hline Experiments on MS2-5 & & & & & & & \\
\hline Specimen & $\# 1$ & $\# 2$ & $\# 3$ & $\# 4$ & $\# 5$ & $\# 6$ & $\# 7$ \\
Bottom & 24 & 18 & 28 & 29 & 32 & 18 & 25 \\
Middle & 34 & 13 & 20 & 21 & 34 & 15 & 23 \\
Top & 21 & 18 & 25 & 24 & 30 & 26 & 24 \\
\hline \hline Experiments on MS2-10 & & & & & & & \\
\hline Specimen & $\# 1$ & $\# 2$ & $\# 3$ & $\# 4$ & $\# 5$ & $\# 6$ & $\# 7$ \\
Bottom & 67 & 31 & 21 & 25 & 37 & 38 & 37 \\
Middle & 47 & 39 & 27 & 38 & 40 & 30 & 37 \\
Top & 37 & 34 & 34 & 40 & 49 & 46 & 40 \\
\hline
\end{tabular}


Table 6: Numbers of the Fibers Bridging the Cracks in Three Divisions of the Cross Section

\begin{tabular}{|c|c|c|c|c|c|c|c|}
\hline \multicolumn{8}{|c|}{ Numerical Simulation on SF-20 } \\
\hline Specimen & $\# 1$ & $\# 2$ & $\# 3$ & $\# 4$ & $\# 5$ & $\# 6$ & $\# 7$ \\
\hline Bottom & 17 & 12 & 10 & 16 & 15 & 8 & 17 \\
\hline Middle & 17 & 11 & 21 & 14 & 15 & 14 & 16 \\
\hline Top & 11 & 13 & 12 & 9 & 5 & 13 & 10 \\
\hline Specimen & $\# 8$ & $\# 9$ & \#10 & \#11 & \#12 & \#13 & \#14 \\
\hline Bottom & 10 & 11 & 28 & 21 & 21 & 19 & 12 \\
\hline Middle & 18 & 15 & 9 & 18 & 15 & 20 & 16 \\
\hline Top & 9 & 12 & 14 & 9 & 4 & 12 & 19 \\
\hline \multicolumn{8}{|c|}{ "Numerical Simulation on SF-35 } \\
\hline Specimen & $\# 1$ & $\# 2$ & $\# 3$ & $\# 4$ & $\# 5$ & $\# 6$ & $\# 7$ \\
\hline Bottom & 25 & 31 & 20 & 27 & 29 & 19 & 33 \\
\hline Middle & 22 & 23 & 31 & 26 & 32 & 31 & 37 \\
\hline Top & 22 & 16 & 23 & 27 & 19 & 10 & 16 \\
\hline Specimen & $\# 8$ & $\# 9$ & \#10 & \#11 & \#12 & \#13 & \#14 \\
\hline Bottom & 27 & 28 & 24 & 35 & 25 & 21 & 16 \\
\hline Middle & 26 & 25 & 22 & 27 & 28 & 29 & 29 \\
\hline Top & 15 & 17 & 19 & 19 & 21 & 19 & 20 \\
\hline \multicolumn{8}{|c|}{ "Numerical Simulation on MS2-5 } \\
\hline Specimen & $\# 1$ & $\# 2$ & $\# 3$ & $\# 4$ & $\# 5$ & $\# 6$ & $\# 7$ \\
\hline Bottom & 18 & 25 & 24 & 27 & 26 & 27 & 23 \\
\hline Middle & 26 & 28 & 23 & 29 & 24 & 20 & 22 \\
\hline Top & 12 & 24 & 13 & 22 & 17 & 11 & 20 \\
\hline \multicolumn{8}{|c|}{ Numerical Simulation on MS2-10 } \\
\hline Specimen & $\# 1$ & $\# 2$ & $\# 3$ & $\# 4$ & $\# 5$ & $\# 6$ & $\# 7$ \\
\hline Bottom & 37 & 43 & 37 & 44 & 44 & 43 & 50 \\
\hline Middle & 36 & 33 & 43 & 38 & 40 & 42 & 57 \\
\hline Top & 38 & 40 & 39 & 40 & 33 & 32 & 38 \\
\hline
\end{tabular}


Table 7: Fracture Process Zone Features at CMOD $=0.1 \mathrm{~mm}$ and CMOD $=1.0 \mathrm{~mm}$

\begin{tabular}{lcccc}
\hline \hline & CMOD $[\mathrm{mm}]$ & FPZ Width $[\mathrm{mm}]$ & Number of Facets & Total Power $[\mathrm{J} / \mathrm{s}]$ \\
\hline Plain Concrete & 0.1 & 75.5 & 6050 & 93.3 \\
Plain Concrete & 1.0 & 36.1 & 1934 & 36.9 \\
SF-20 & 0.1 & 75.6 & 5566 & 85.1 \\
SF-20 & 1.0 & 76.2 & 4876 & 90.1 \\
SF-35 & 0.1 & 80.8 & 6866 & 79.7 \\
SF-35 & 1.0 & 73.8 & 6170 & 126.4 \\
MS2-5 & 0.1 & 84.3 & 6664 & 99.4 \\
MS2-5 & 1.0 & 38.4 & 2648 & 56.7 \\
MS2-10 & 0.1 & 82.2 & 6180 & 89.7 \\
MS2-10 & 1.0 & 65.8 & 3108 & 43.4 \\
\hline
\end{tabular}


a)

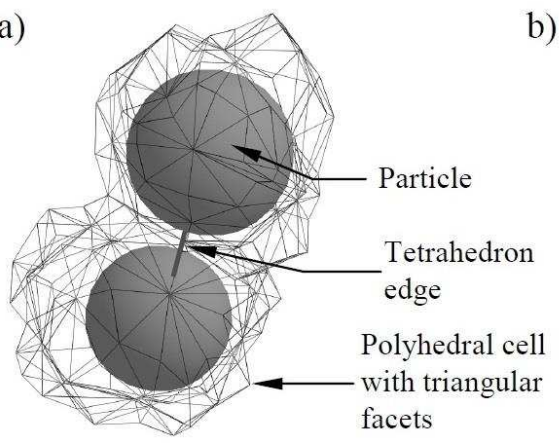

b)

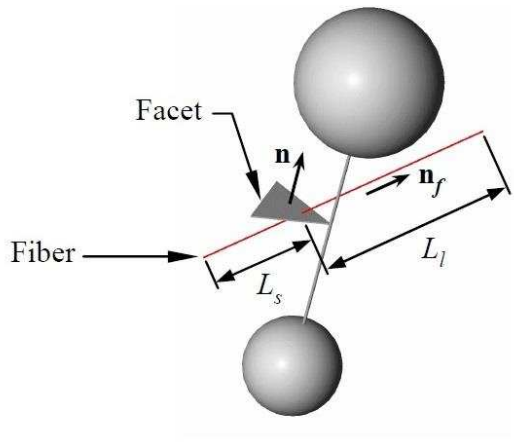

Figure 1: LDPM simulation for concrete mesostructure: (a) Two adjacent particles; (b) Facet with intersecting fiber. 


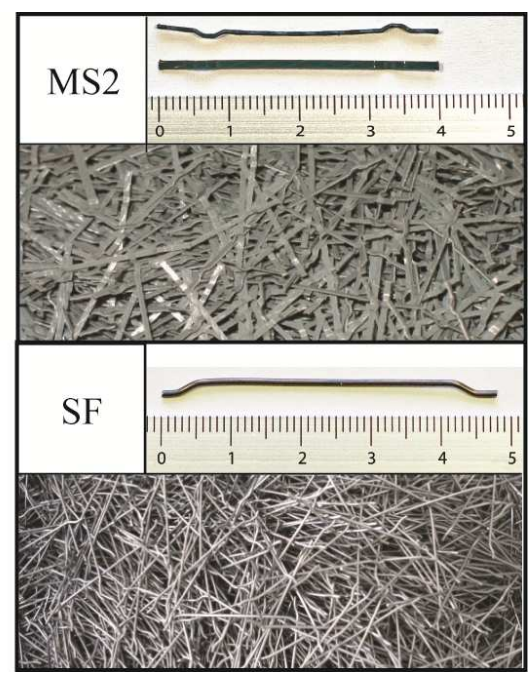

Figure 2: Fibers used in the experiments. MS2 represents synthetic fibers, and SF represents steel fibers. Scales are in $\mathrm{cm}$. 
(a)

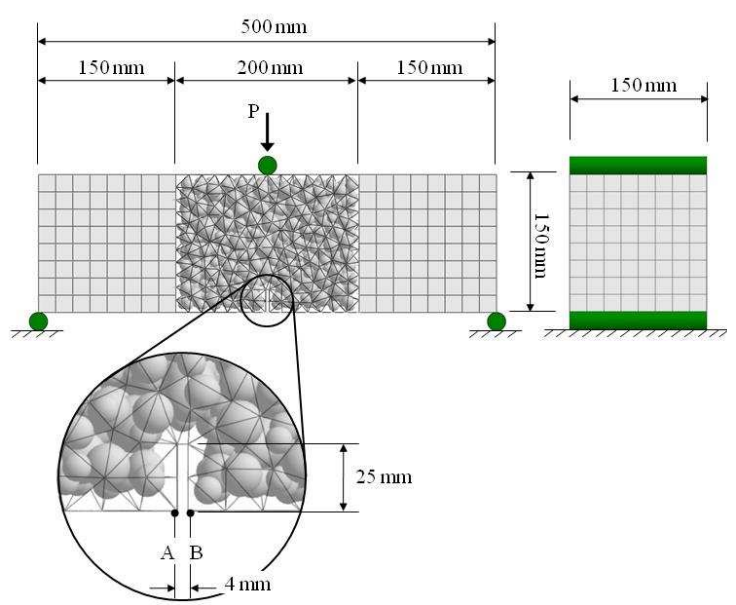

(c)

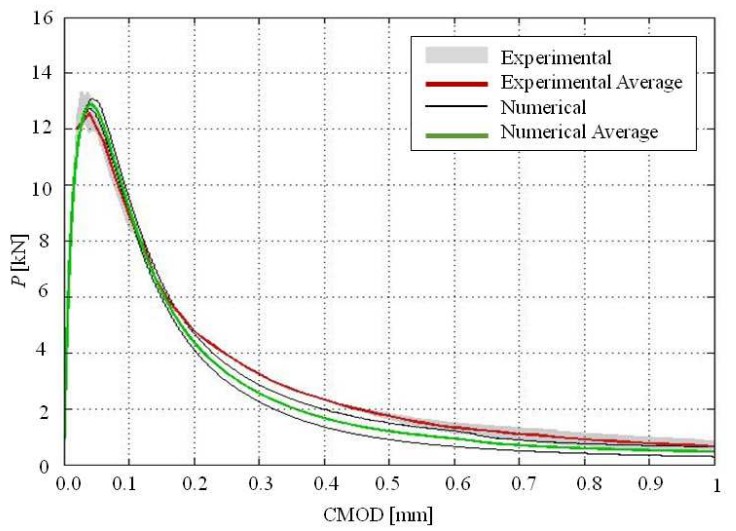

(b)

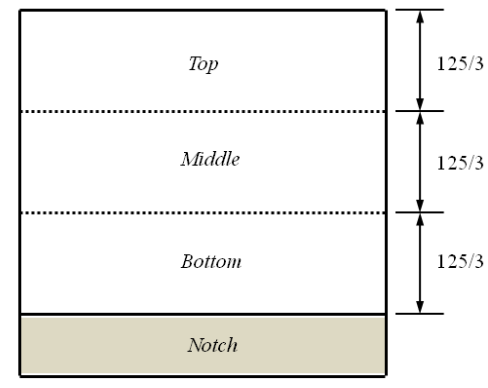

(d)

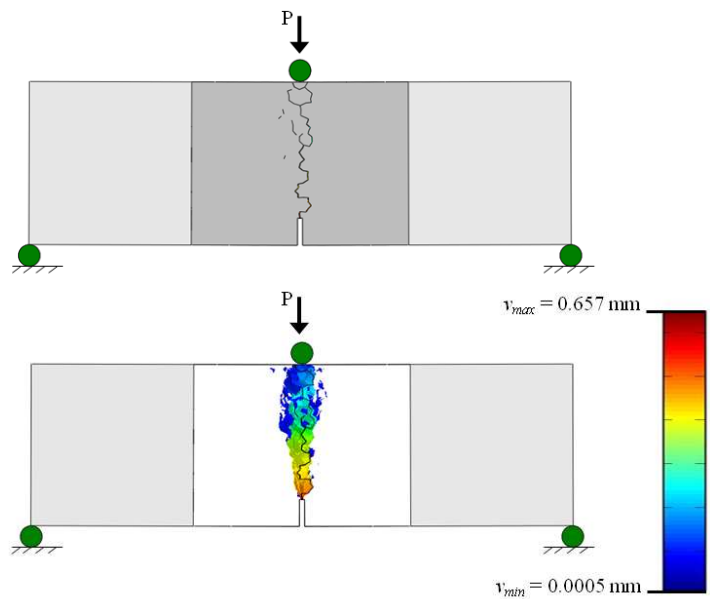

Figure 3: LDPM simulation for plain concrete: (a) Specimen geometry and discretization in numerical simulation; (b) Three divisions of the cross section; (c) Load vs CMOD curve; and (d) Meso-scale crack patterns at CMOD $=0.8 \mathrm{~mm}$. The experimental curve (red line) represents the average of two experimental tests, and the numerical curve (green line) represents the average response from two distinct meso-structural models. 
(a)

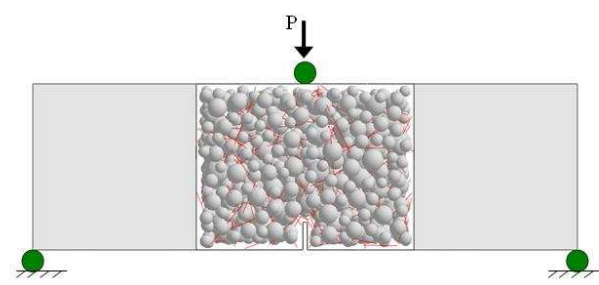

(b)

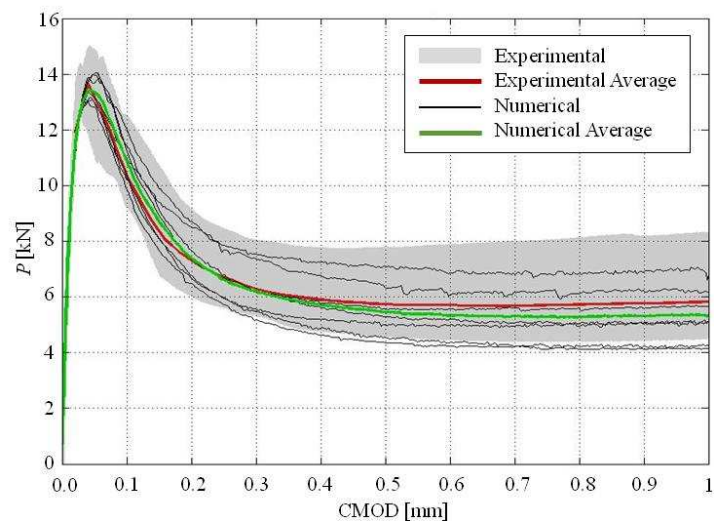

(c)
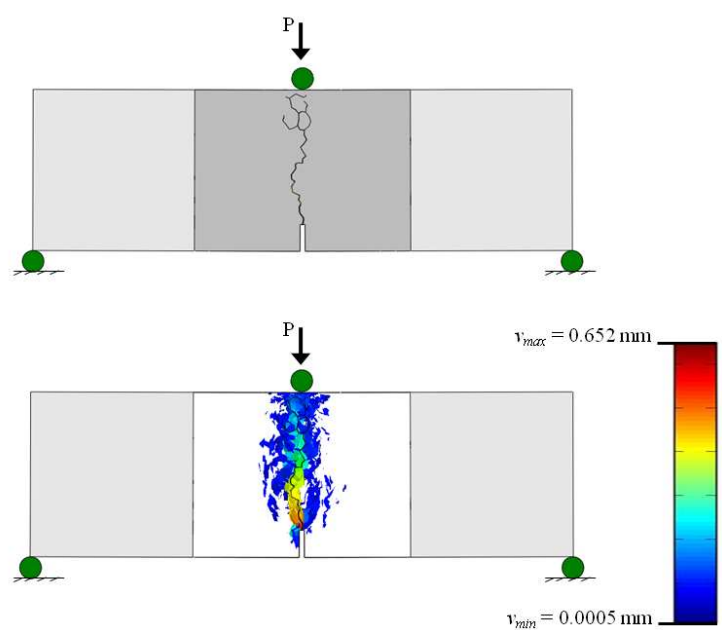

Figure 4: LDPM simulation for steel fiber reinforced concrete SF-20 with $V_{f}=0.26 \%$ : (a) Specimen geometry and discretization in numerical simulation; (b) Load vs CMOD curve; and (c) Meso-scale crack patterns at CMOD $=0.8 \mathrm{~mm}$. The experimental curve (red line) represents the average of seven experimental tests, and the numerical curve (green line) represents the average response from seven distinct meso-structural models. 
(a)

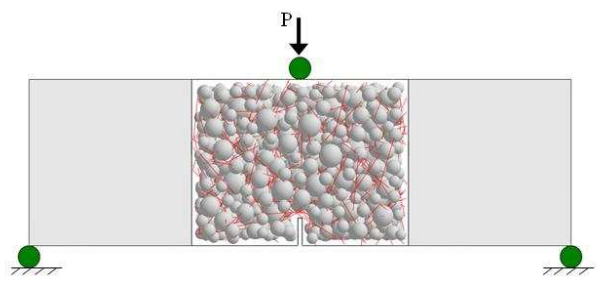

(b)

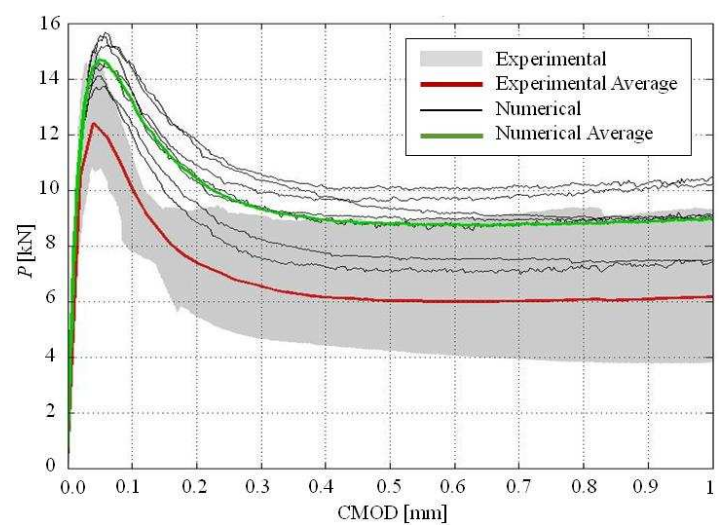

(c)
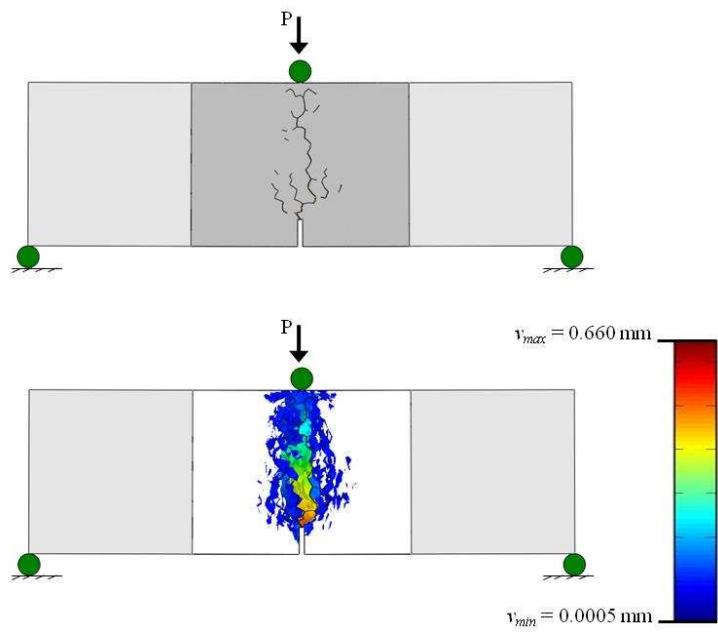

Figure 5: LDPM simulation for steel fiber reinforced concrete SF-35 with $V_{f}=0.45 \%$ : (a) Specimen geometry and discretization in numerical simulation; (b) Load vs CMOD curve; and (c) Meso-scale crack patterns at CMOD $=0.8 \mathrm{~mm}$. The experimental curve (red line) represents the average of seven experimental tests, and the numerical curve (green line) represents the average response from seven distinct meso-structural models. 
(a)

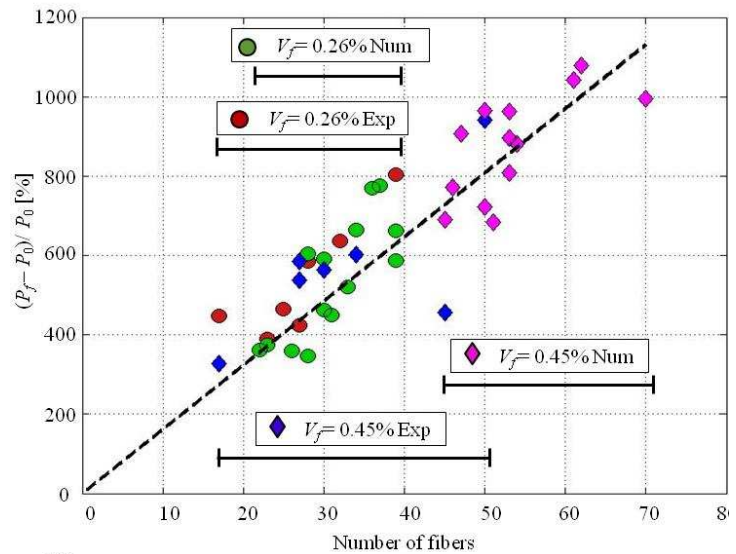

(b)

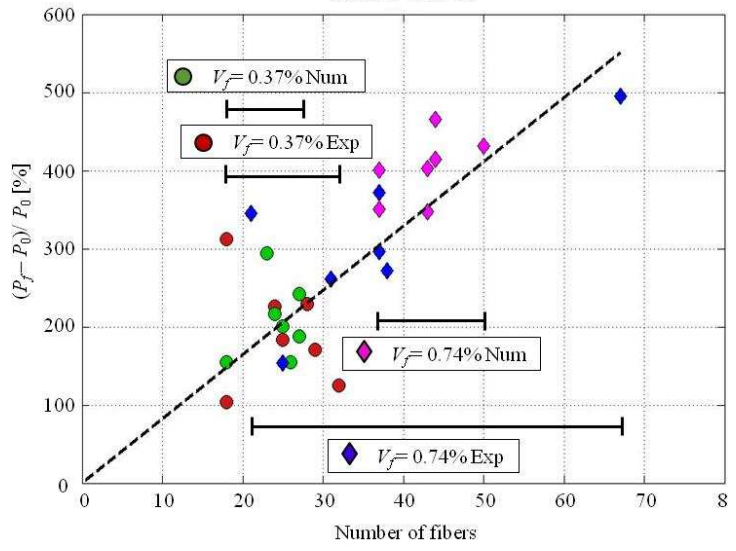

(c)

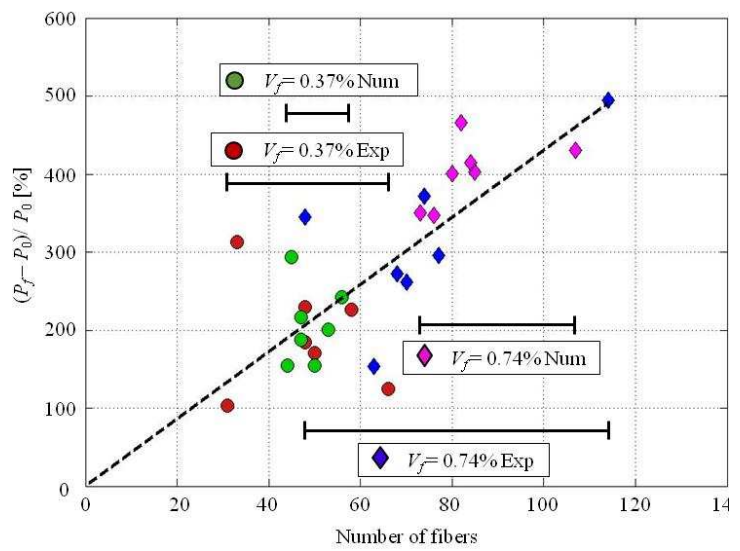

Figure 6: (a) Load increment versus number of active fibers in Bottom +Middle of the steel fiber reinforced concrete; (b) Load increment versus number of active fibers in Bottom of the synthetic fiber reinforced concrete; (c) Load increment versus number of active fibers in Bottom + Middle of the synthetic fiber reinforced concrete. 
(a)
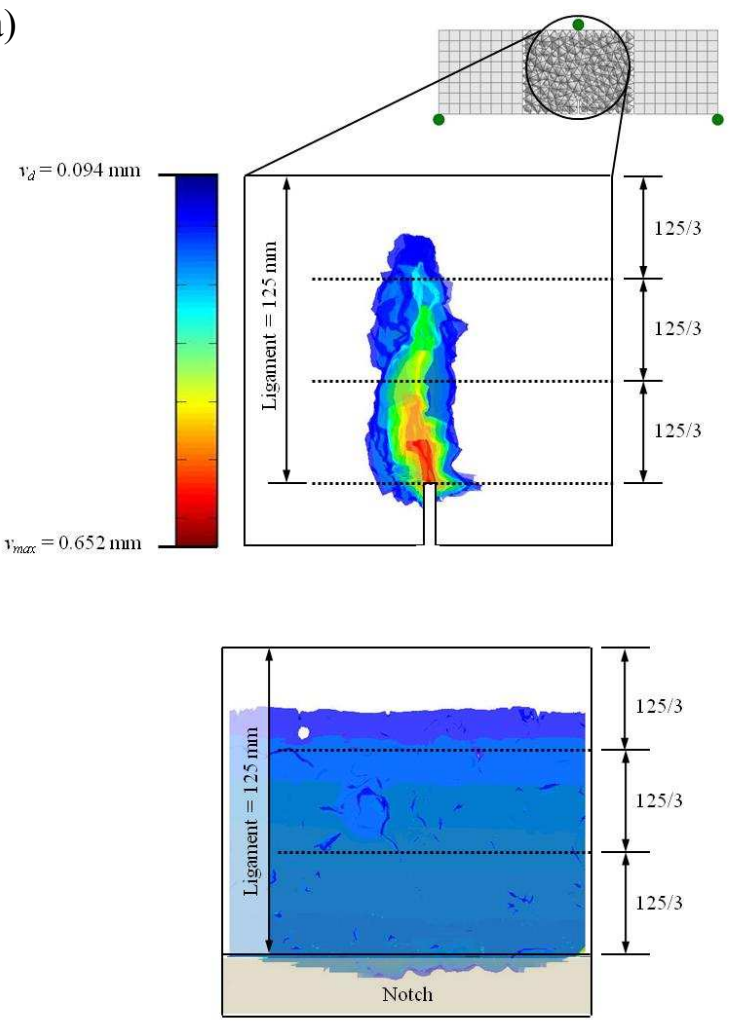

(b)
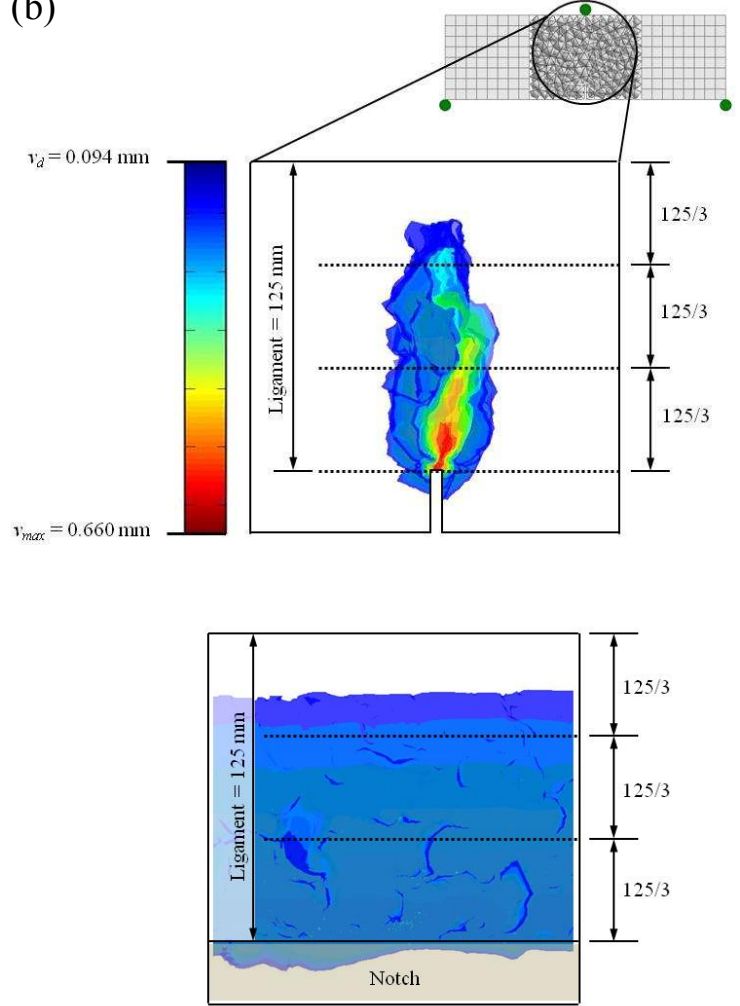

Figure 7: Crack opening isosurfaces at $\mathrm{CMOD}=0.8 \mathrm{~mm}$ for steel fiber reinforced concrete: (a) $V_{f}=0.26 \%$; (b) $V_{f}=0.45 \%$. 
(a)

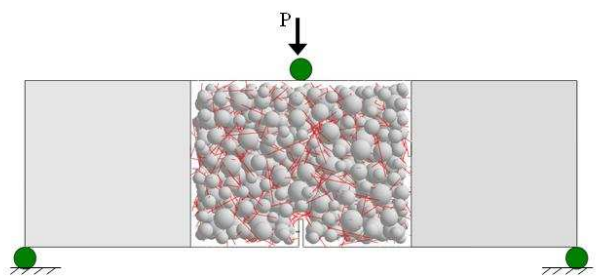

(b)

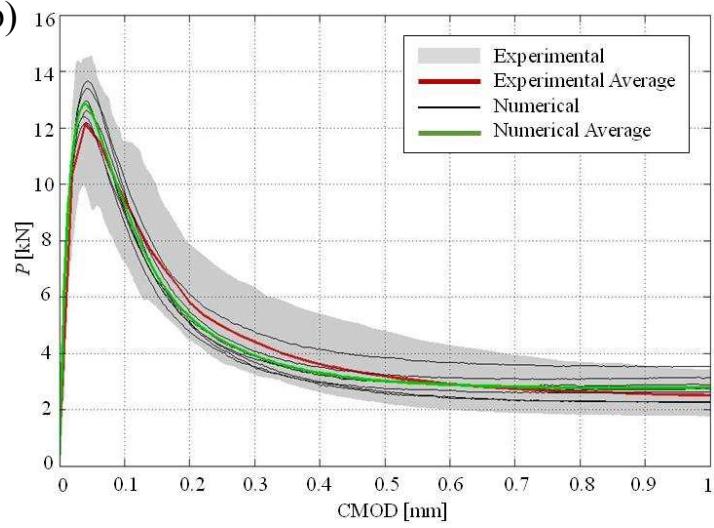

(c)
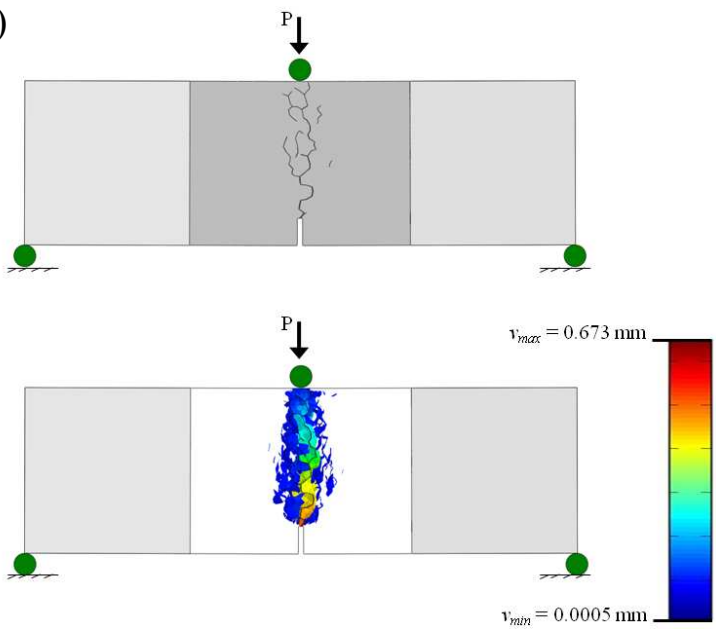

Figure 8: LDPM simulation for synthetic fiber reinforced concrete MS2-5 with $V_{f}=0.37 \%$ : (a) Specimen geometry and discretization in numerical simulation; (b) Load vs CMOD curve; and (c) Meso-scale crack patterns at CMOD $=0.8 \mathrm{~mm}$. The experimental curve (red line) represents the average of seven experimental tests, and the numerical curve (green line) represents the average response from seven distinct meso-structural models. 
(a)

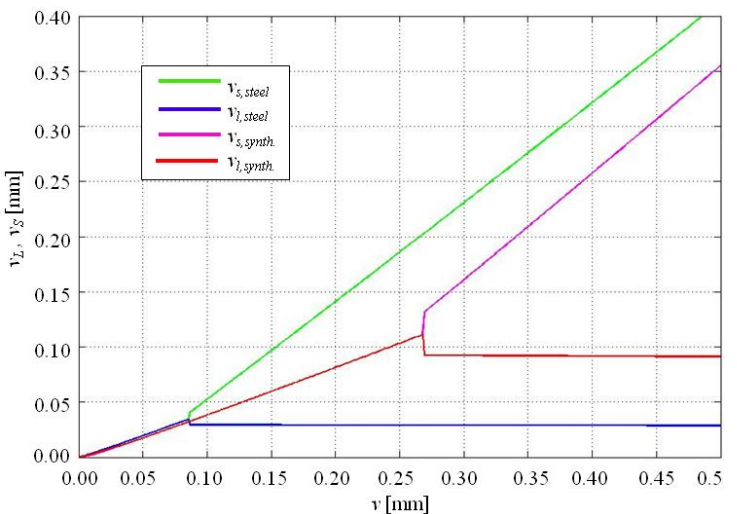

(b)

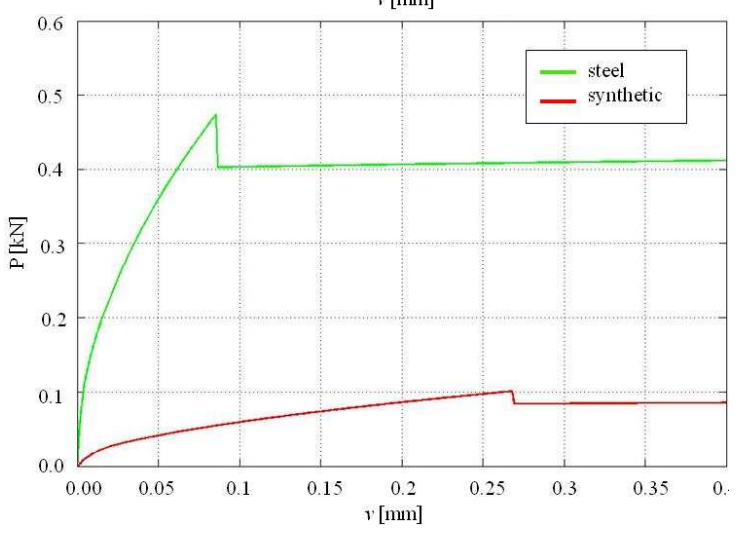

Figure 9: Single fiber two way pull-out test: (a) Relationship between fiber slippage and crack opening for steel and synthetic fibers; (b) Relationship between load and crack opening for steel and synthetic fibers. 
(a)

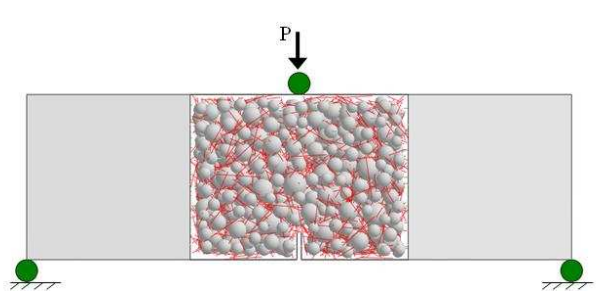

(b)

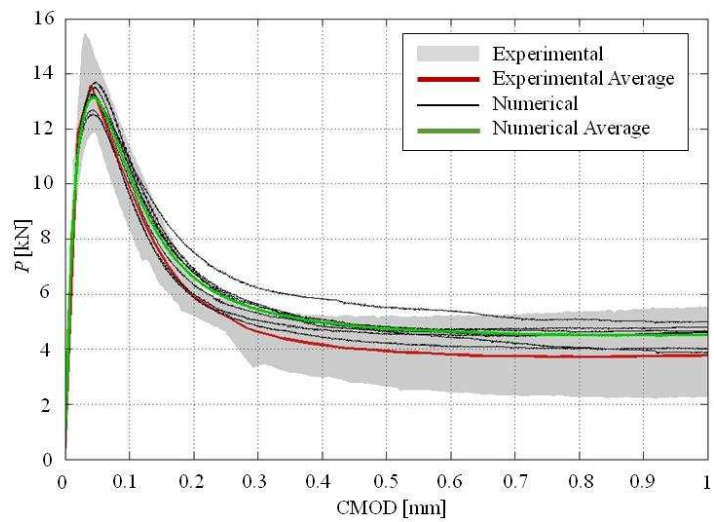

(c)
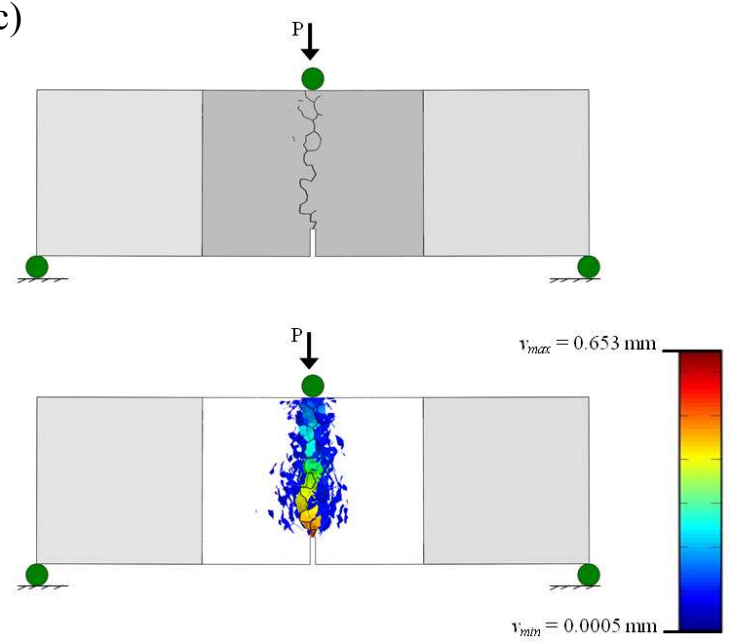

Figure 10: LDPM simulation for synthetic fiber reinforced concrete MS2-10 with $V_{f}=$ 0.74\%: (a) Specimen geometry and discretization in numerical simulation; (b) Load vs CMOD curve; and (c) Meso-scale crack patterns at CMOD $=0.8 \mathrm{~mm}$. The experimental curve (red line) represents the average of seven experimental tests, and the numerical curve (green line) represents the average response from seven distinct meso-structural models. 

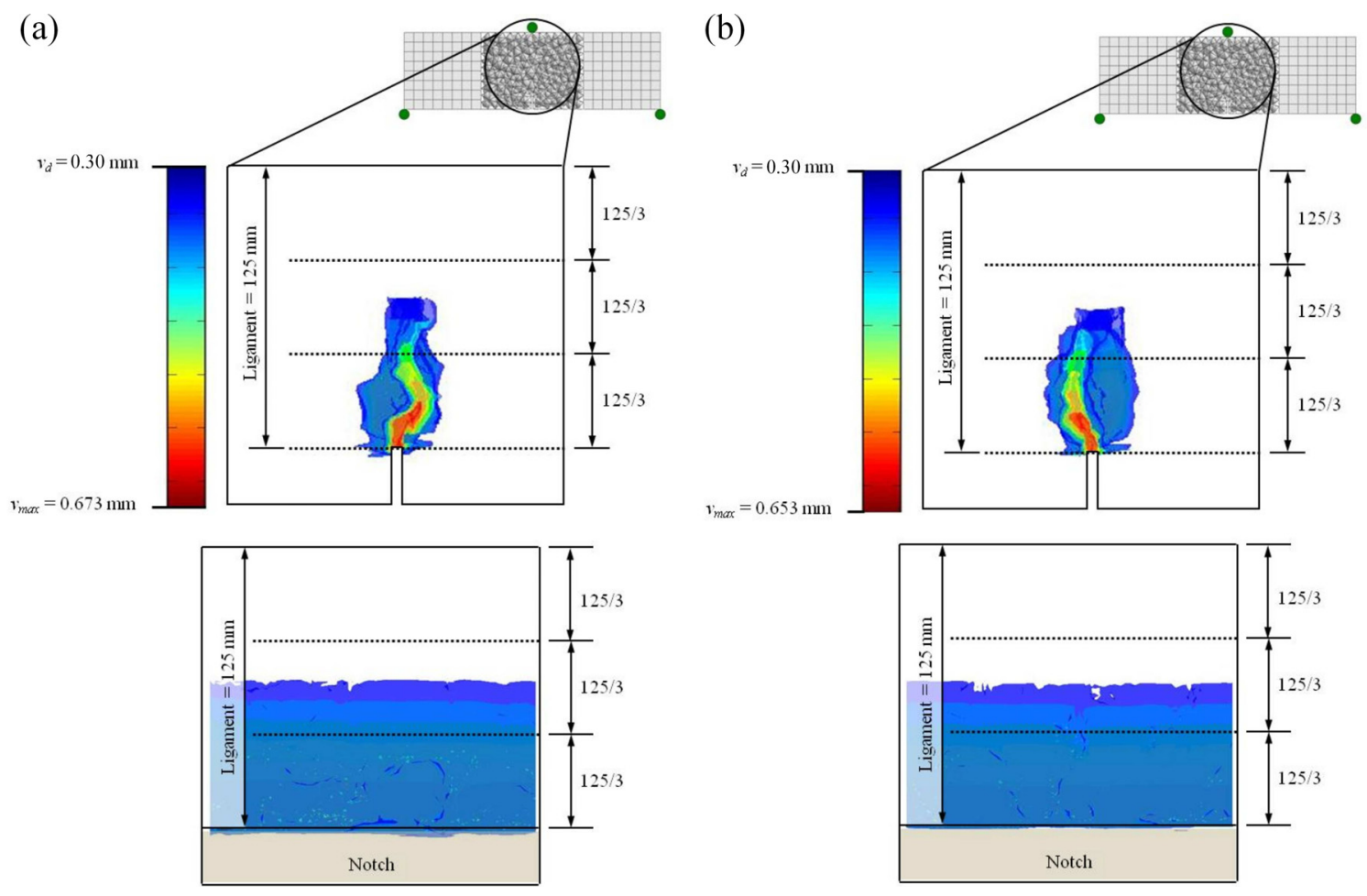

Figure 11: Crack opening isosurfaces at $\mathrm{CMOD}=0.8 \mathrm{~mm}$ for synthetic fiber reinforced concrete: (a) $V_{f}=0.37 \%$; (b) $V_{f}=0.74 \%$. 

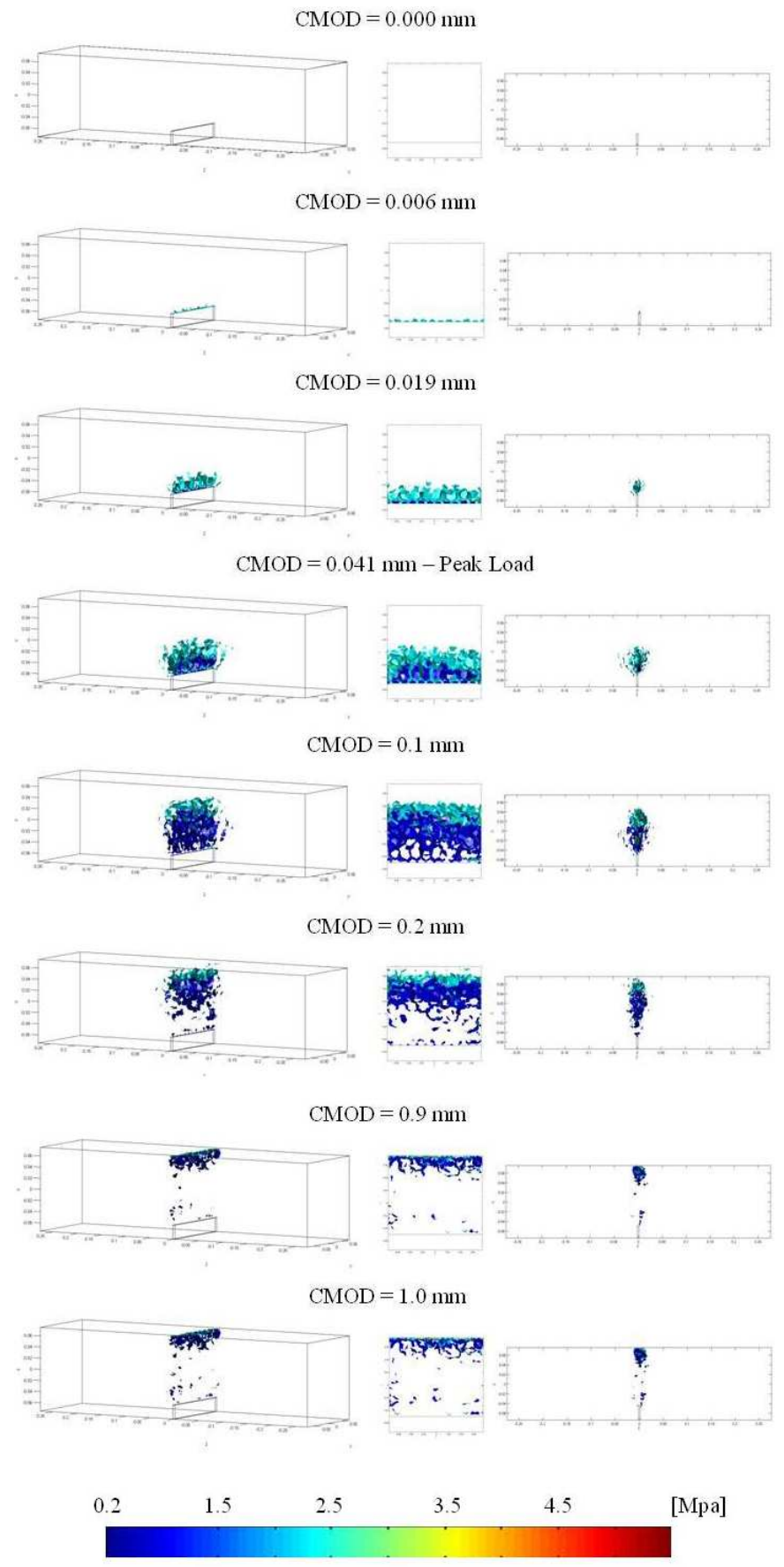

Figure 12: Fracture process zone for plain concrete. 

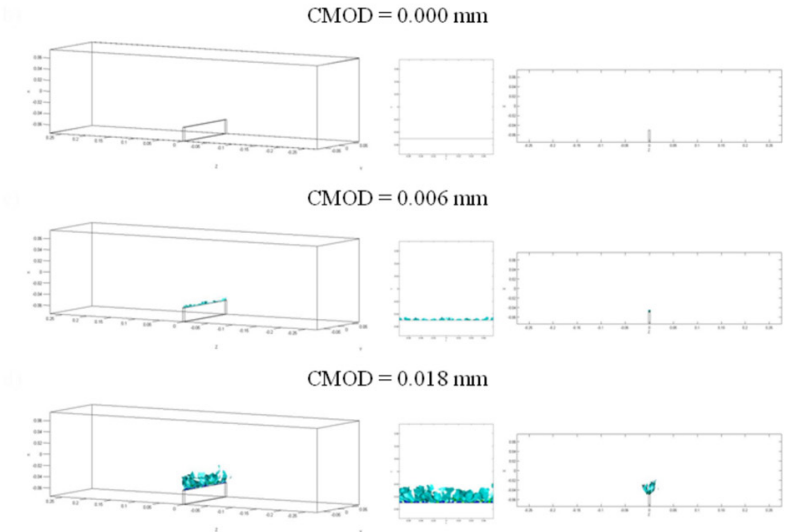

$\mathrm{CMOD}=0.039 \mathrm{~mm}-$ Peak Load

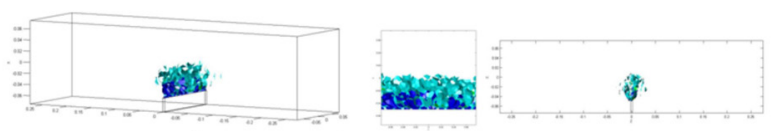

$\mathrm{CMOD}=0.1 \mathrm{~mm}$

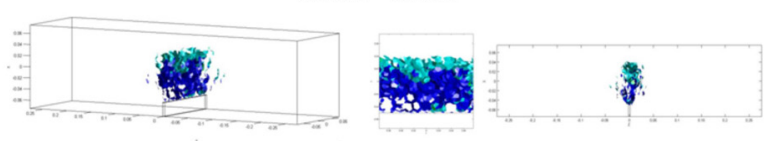

$\mathrm{CMOD}=0.2 \mathrm{~mm}$
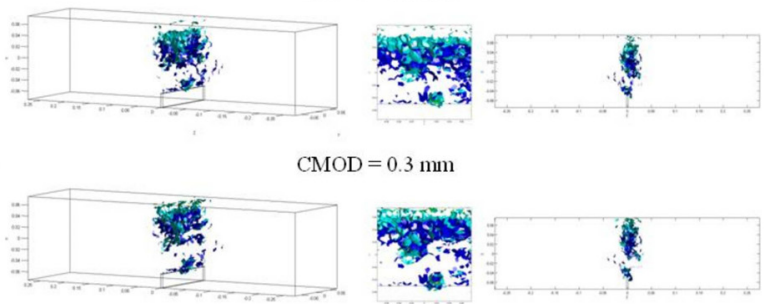

$\mathrm{CMOD}=0.4 \mathrm{~mm}$

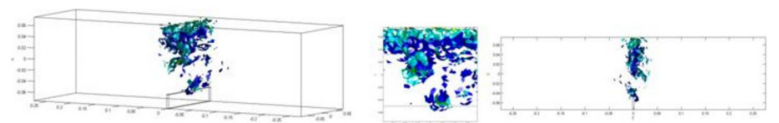

$\mathrm{CMOD}=0.5 \mathrm{~mm}$
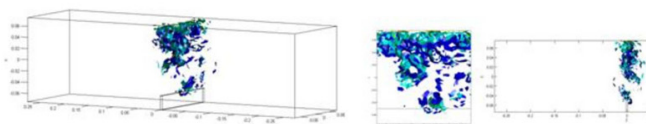

$\mathrm{CMOD}=0.6 \mathrm{~mm}$
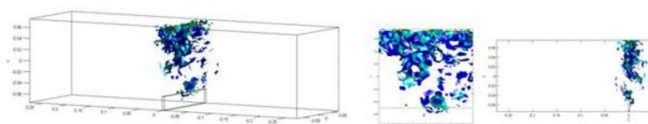

$\mathrm{CMOD}=0.7 \mathrm{~mm}$
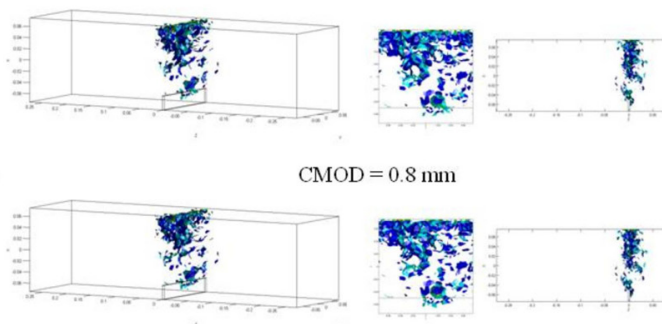

$\mathrm{CMOD}=0.9 \mathrm{~mm}$
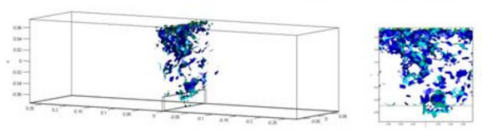

零

$\mathrm{CMOD}=1.0 \mathrm{~mm}$
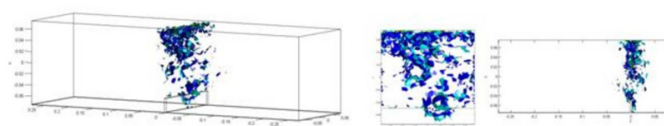

0.2

1.5

2.5

3.5

4.5

[Mpa]

Figure 13: Fracture process zone for steel fiber reinforced concrete with $V_{f}=0.26 \%$. 


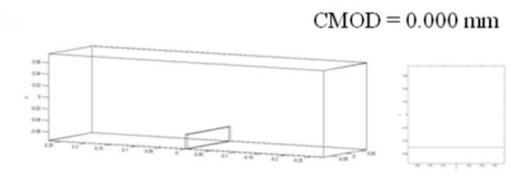

$\mathrm{CMOD}=0.006 \mathrm{~mm}$
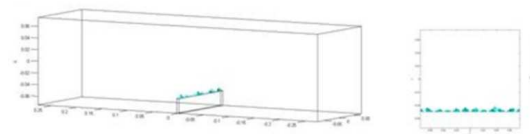

$\mathrm{CMOD}=0.019 \mathrm{~mm}$
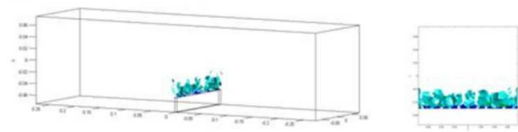

$\mathrm{CMOD}=0.039 \mathrm{~mm}-$ Peak Loa
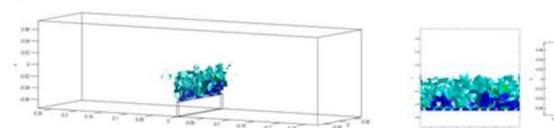

桴

$\mathrm{CMOD}=0.1 \mathrm{~mm}$
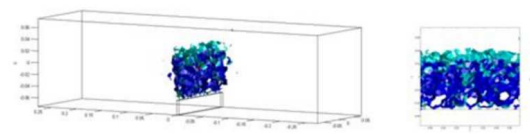

$\mathrm{CMOD}=0.2 \mathrm{~mm}$
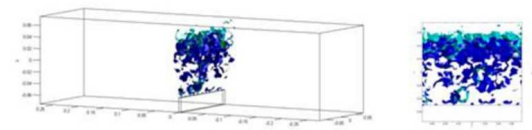

$\mathrm{CMOD}=0.3 \mathrm{~mm}$
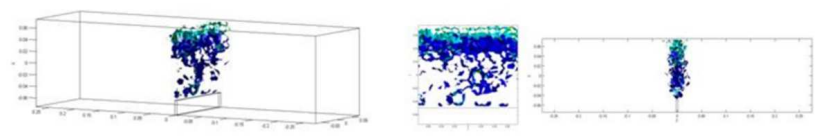

$\mathrm{CMOD}=0.4 \mathrm{~mm}$
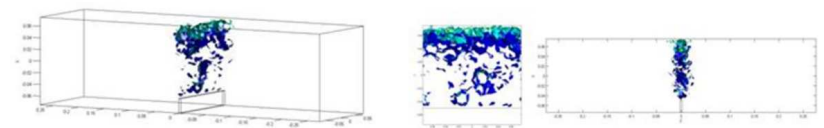

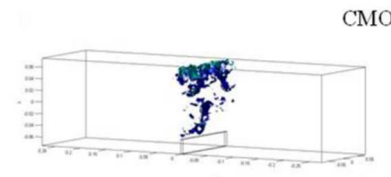

$\mathrm{MOD}=0.5 \mathrm{~mm}$

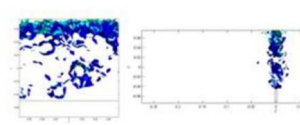

$\mathrm{CMOD}=0.6 \mathrm{~mm}$

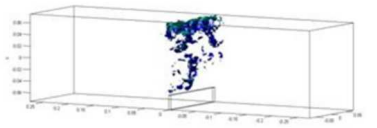

toses? s

$\mathrm{CMOD}=0.7 \mathrm{~mm}$
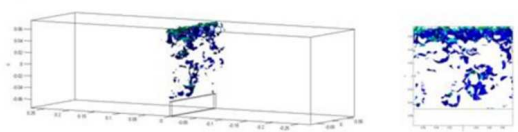

震

$\mathrm{CMOD}=0.8 \mathrm{~mm}$

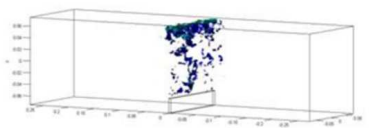

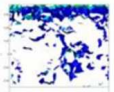

零

$\mathrm{CMOD}=0.9 \mathrm{~mm}$

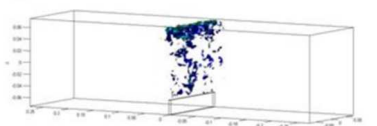

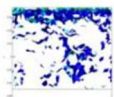

零

$\mathrm{CMOD}=1.0 \mathrm{~mm}$

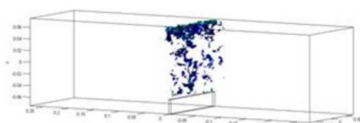

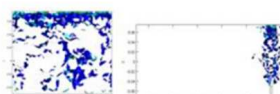

\section{2}

1.5

2.5

3.5

4.5

[Mpa]

Figure 14: Fracture process zone for synthetic fiber reinforced concrete with $V_{f}=0.74 \%$. 
(a)

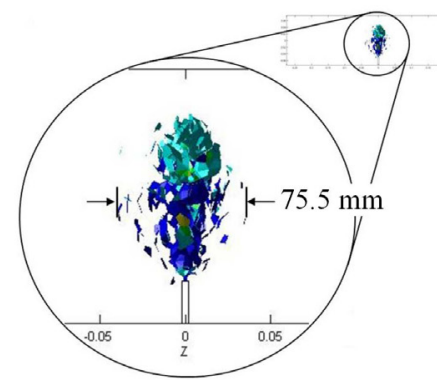

(c)

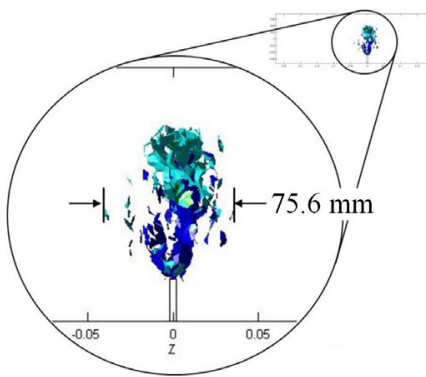

(e)

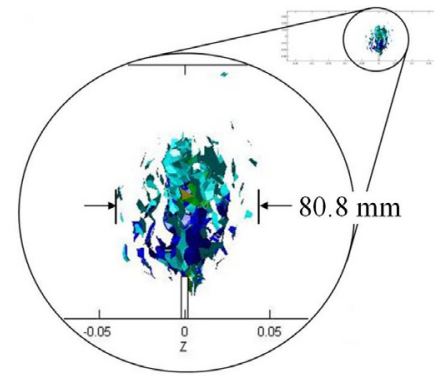

$(\mathrm{g})$

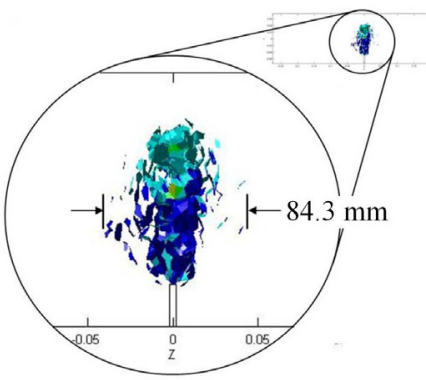

(i)

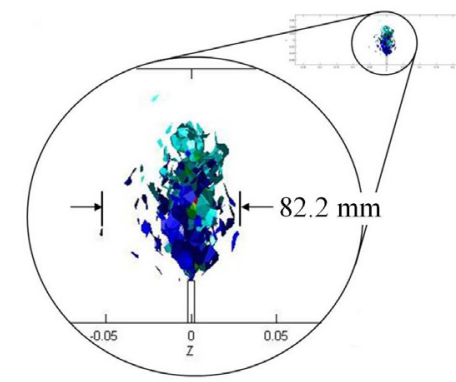

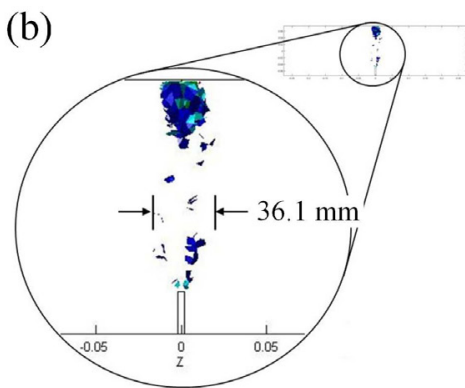

(d)

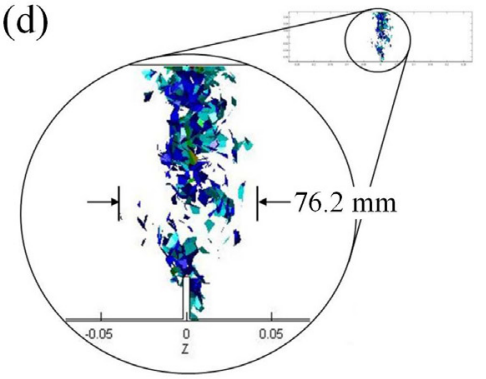

(f)

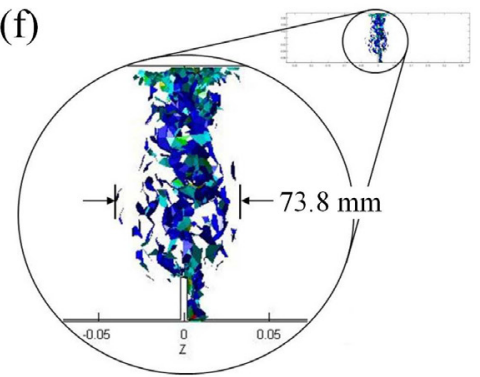

(h)

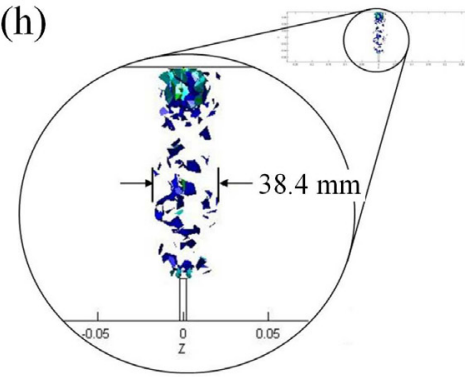

(j)

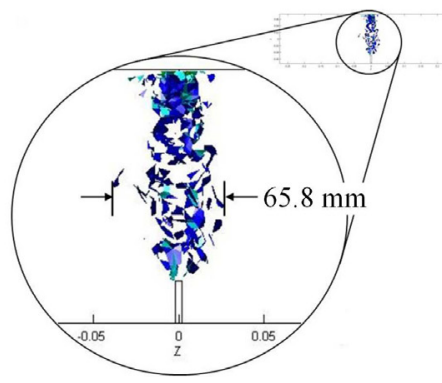

51

Figure 15: Fracture process zone for (a) plain concrete at CMOD $=0.1 \mathrm{~mm}$, and (b) at $\mathrm{CMOD}=1.0 \mathrm{~mm}$; (c) steel fiber reinforced concrete with $V_{f}=0.26 \%$ at CMOD $=0.1$ $\mathrm{mm}$, and (d) at CMOD $=1.0 \mathrm{~mm}$; (e) steel fiber reinforced concrete with $V_{f}=0.45 \%$ at $\mathrm{CMOD}=0.1 \mathrm{~mm}$, and $(\mathrm{f})$ at $\mathrm{CMOD}=1.0 \mathrm{~mm} ;(\mathrm{g})$ synthetic fiber reinforced concrete with $V_{f}=0.37 \%$ at CMOD $=0.1 \mathrm{~mm}$, and $(\mathrm{h})$ at $\mathrm{CMOD}=1.0 \mathrm{~mm}$; (i) synthetic fiber reinforced concrete with $V_{f}=0.74 \%$ at CMOD $=0.1 \mathrm{~mm}$, and $(\mathrm{j})$ at $\mathrm{CMOD}=1.0 \mathrm{~mm}$. 


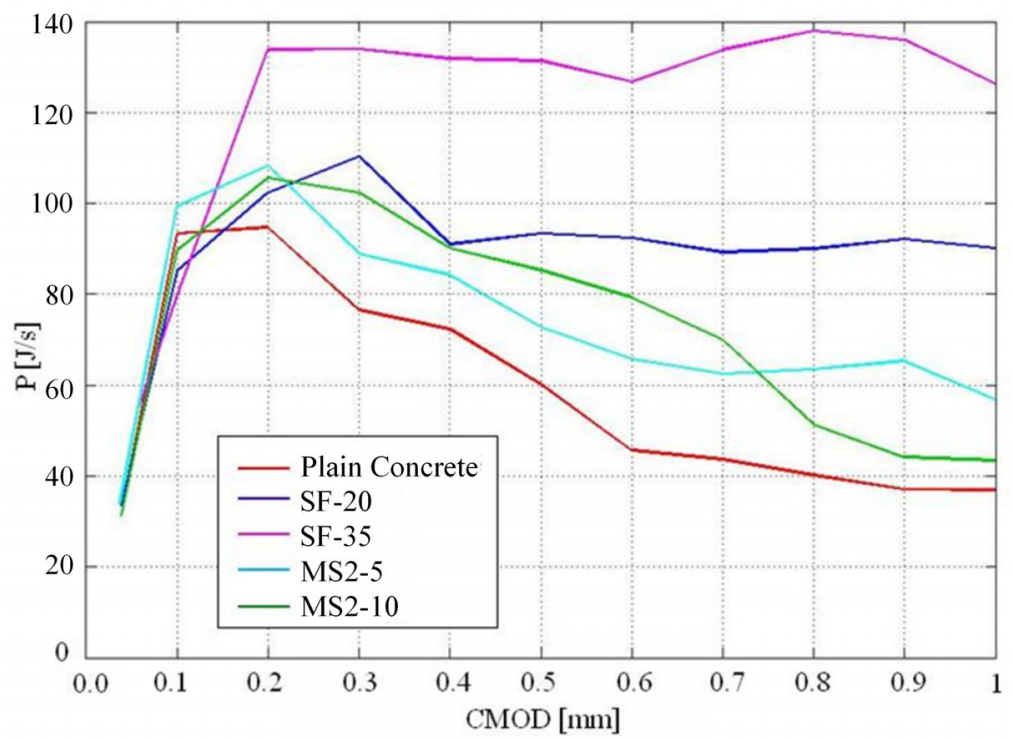

Figure 16: The trend of the total power during the test for the five different analyzed specimens. 

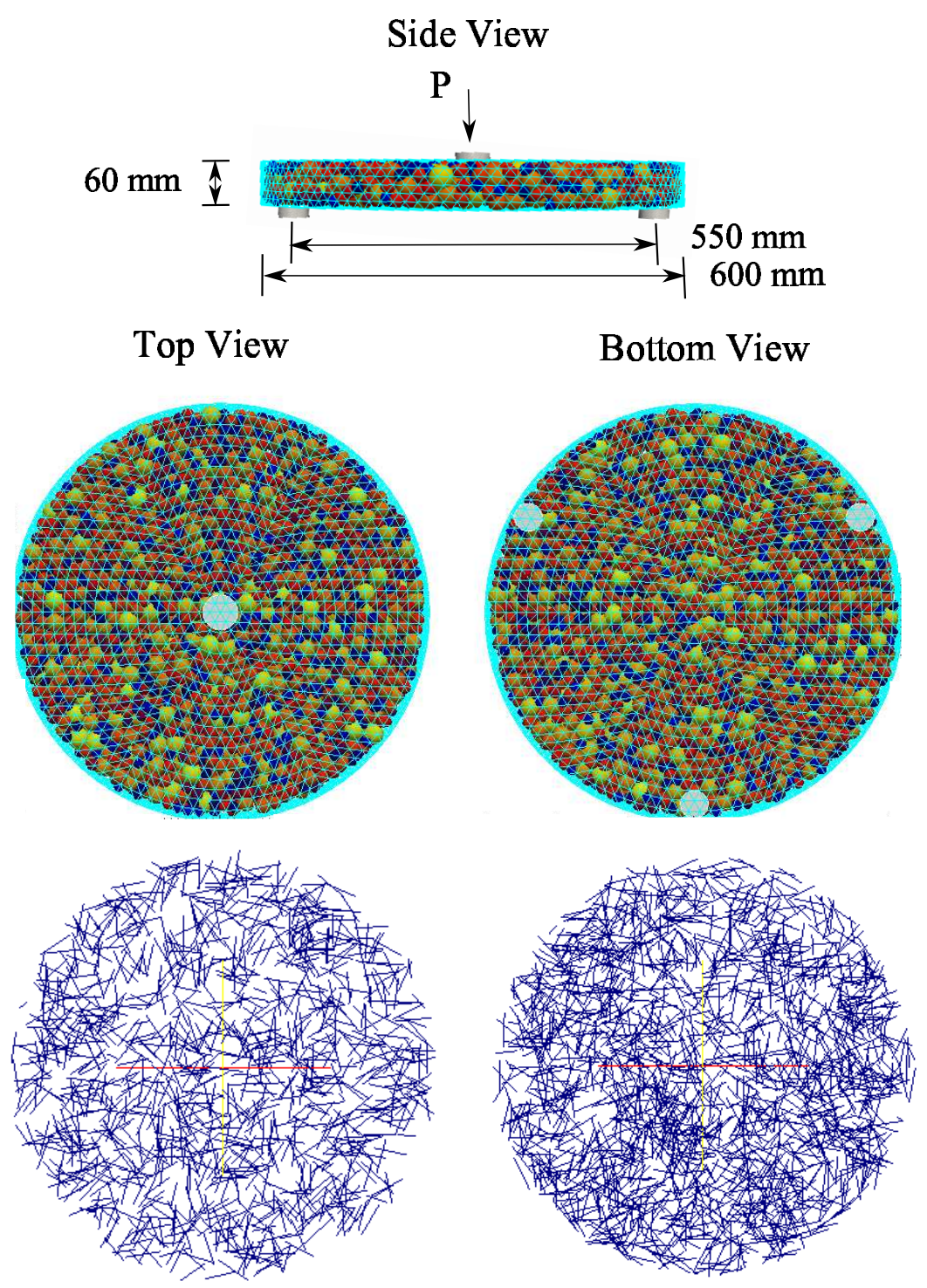

Figure 17: LDPM simulation for round panel test on small specimens. This figures shows the specimen geometry and discretization for plain concrete and steel fiber reinforced concrete with $V_{f}=0.26 \%$ and $V_{f}=0.39 \%$. 
(a)

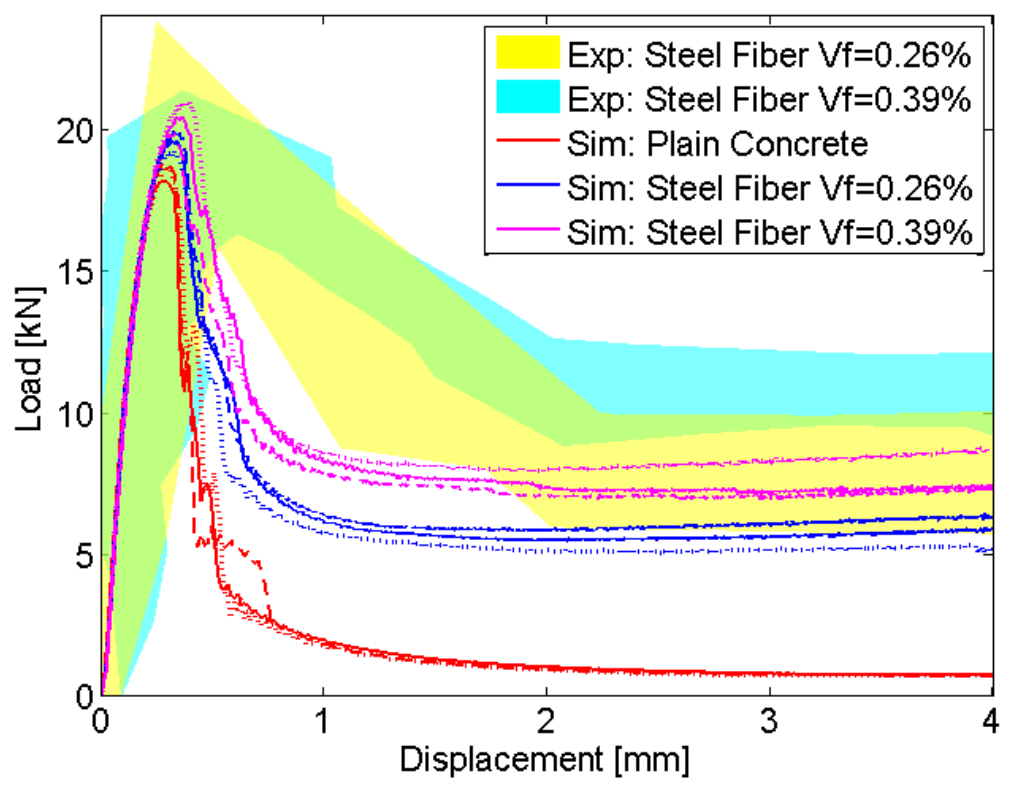

(b)

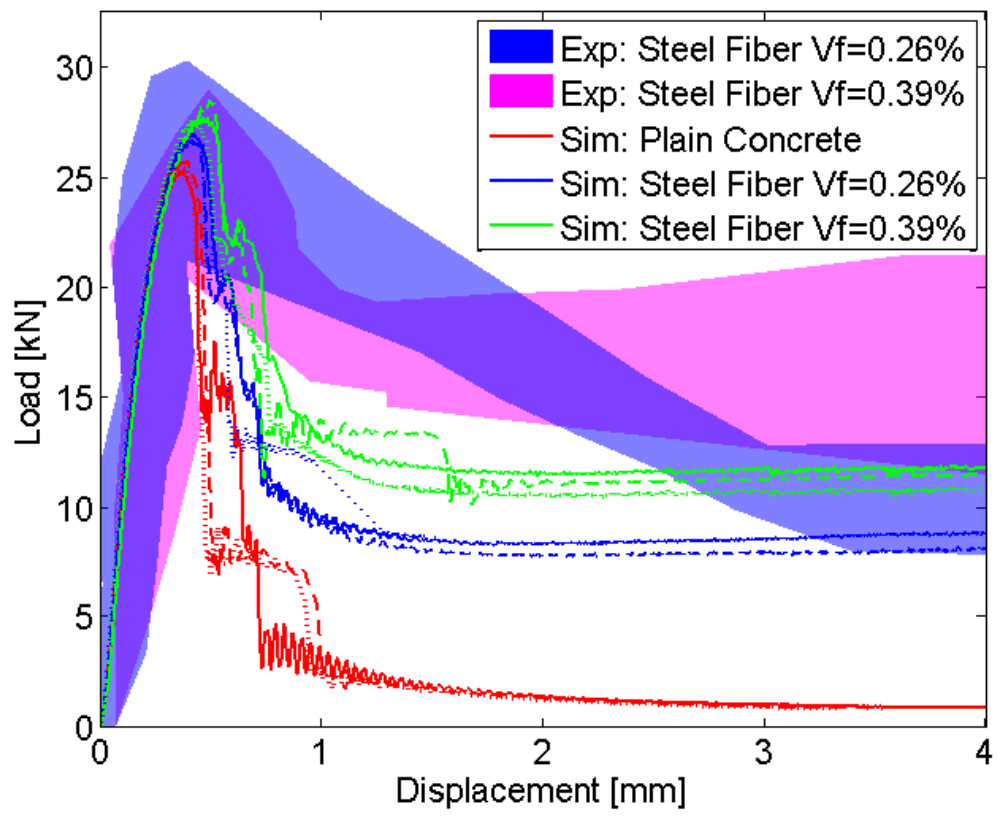

Figure 18: Experimental data and numerical simulation results for the relationship between load and deflection (obtained from three numerical specimens in each case) for different kinds of fiber reinforcement, together with the curves for plain concrete: (a) Small round panel test; (b) Large round panel test. 
(a)
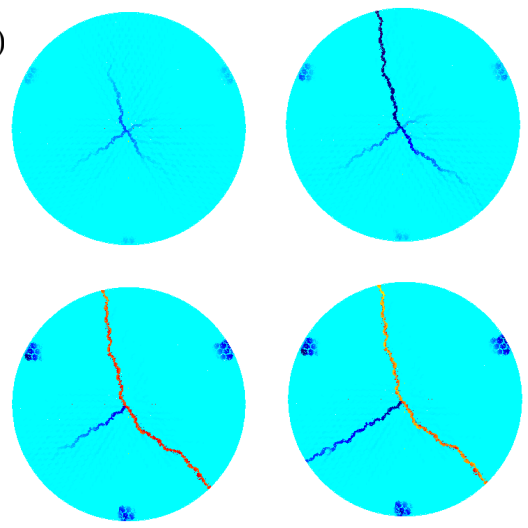

(b)

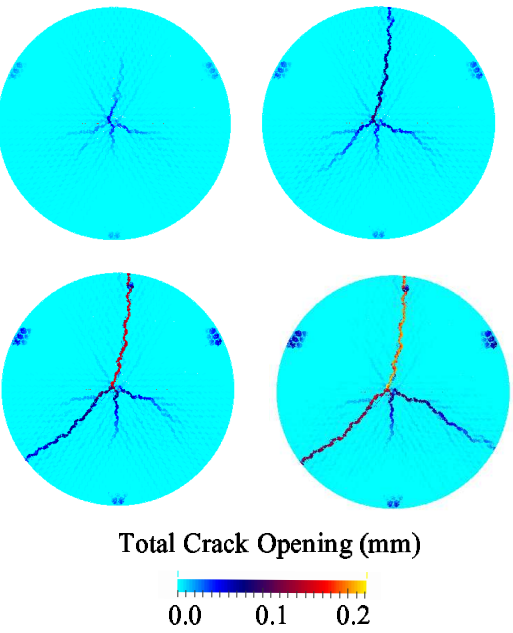

Figure 19: LDPM simulation for round panel test on small specimens. This figures shows the crack propagation through the panel: (a) Plain concrete at $\delta=0.30 \mathrm{~mm}, \delta=0.38$ $\mathrm{mm}, \delta=0.45 \mathrm{~mm}$, and $\delta=0.53 \mathrm{~mm}$; (b) Steel fiber reinforced concrete with $V_{f}=0.39 \%$ at $\delta=0.32 \mathrm{~mm}, \delta=0.45 \mathrm{~mm}, \delta=0.54 \mathrm{~mm}$, and $\delta=0.63 \mathrm{~mm}$. 
(a)
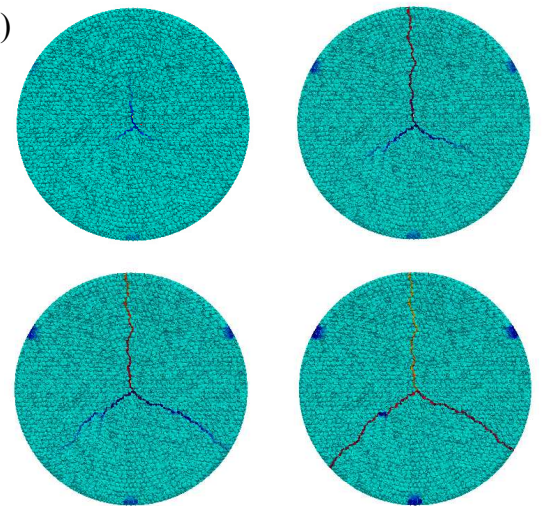

(b)

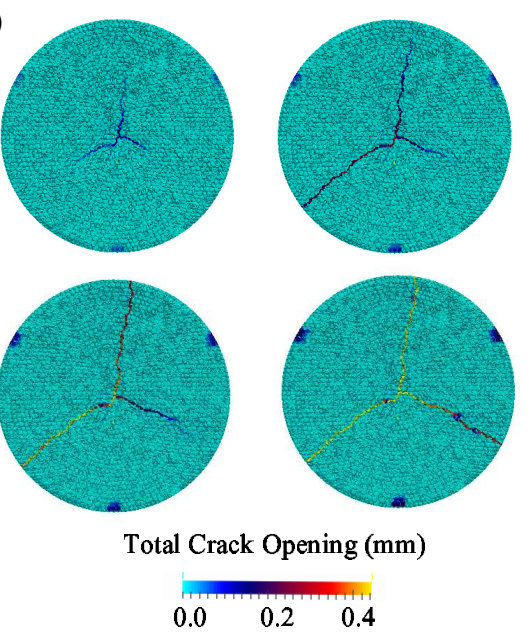

Figure 20: LDPM simulation for round panel test on large specimens. This figures shows the crack propagation through the bottom surface: (a) Plain concrete at $\delta=0.33 \mathrm{~mm}$, $\delta=0.49 \mathrm{~mm}, \delta=0.50 \mathrm{~mm}$, and $\delta=1.10 \mathrm{~mm}$; (b) Steel fiber reinforced concrete with $V_{f}=0.39 \%$ at $\delta=0.48 \mathrm{~mm}, \delta=0.57 \mathrm{~mm}, \delta=1.43 \mathrm{~mm}$, and $\delta=1.90 \mathrm{~mm}$. 

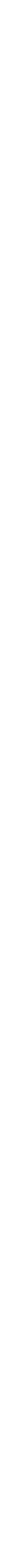

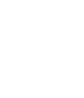
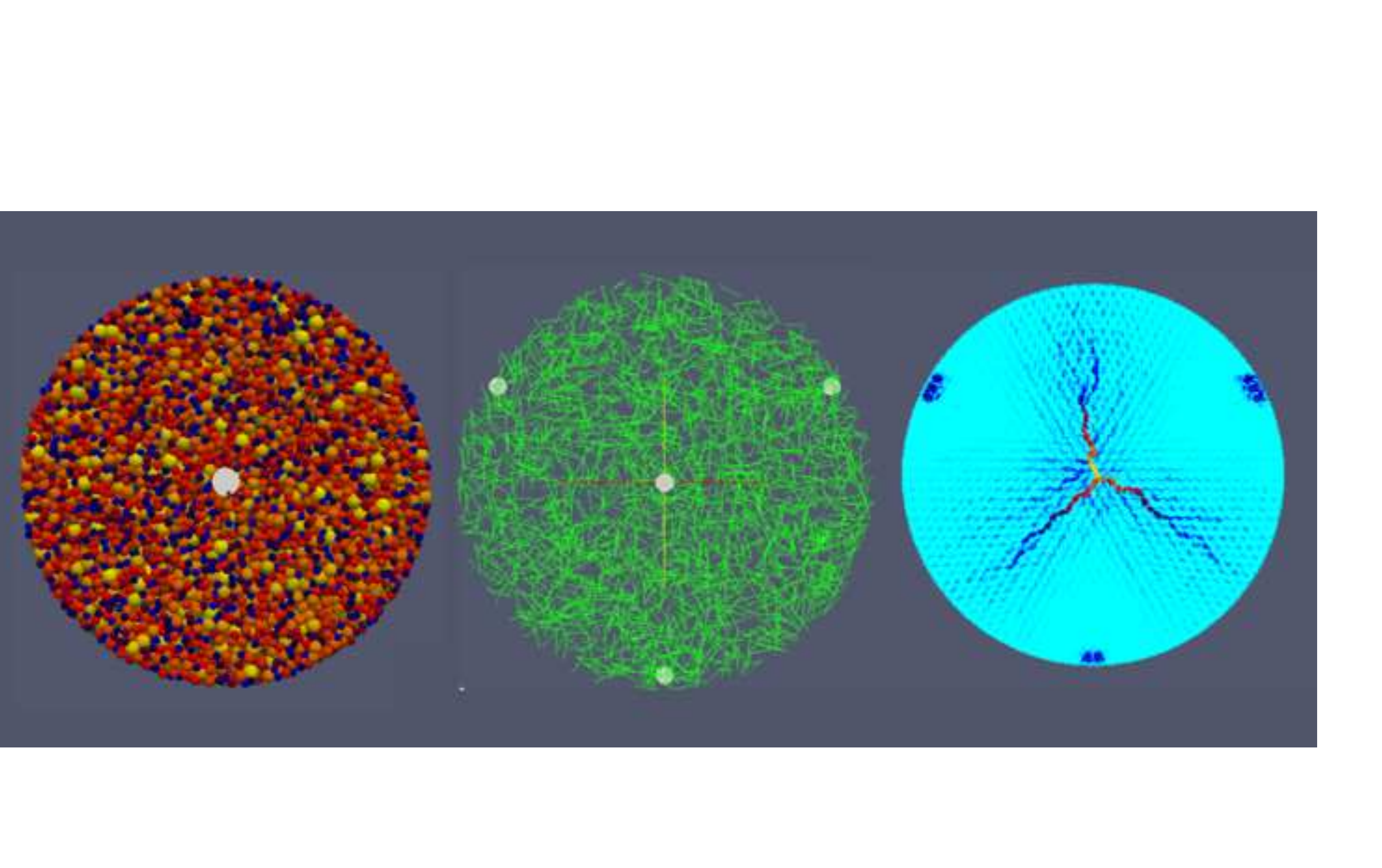

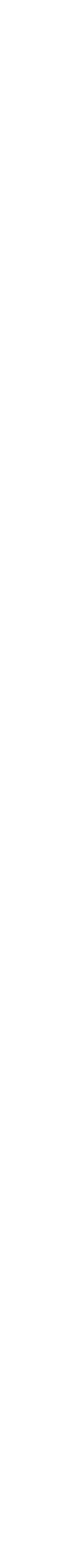

